\title{
Deliberating Collective Decisions
}

\author{
Jimmy Chan* Alessandro Lizzeri ${ }^{\dagger} \quad$ Wing Suen ${ }^{\ddagger} \quad$ Leeat Yariv $^{\$ \uparrow}$
}

August 30, 2016

\begin{abstract}
We present a dynamic model of sequential information acquisition by a heterogeneous committee. At each date agents decide whether to vote to adopt one of two alternatives or continue to collect more information. The process stops when a qualified majority vote for an alternative. Three main insights emerge from our analysis and match an array of stylized facts on committee decision making. First, majority rule is more fragile than super-majority rules to the disproportionate influence of impatient committee members. Second, more diverse preferences, more patient members, or more unanimous decision voting rules lead to lengthier deliberation and more accurate decisions. Last, balanced committees unanimously prefer to delegate deliberation power to a moderate chairman rather than be governed by a rule such as unanimity.
\end{abstract}

JEL Classification: D71, D72, D83

Keywords: sequential likelihood ratio test, optimal stopping, swing voters, collective learning

\footnotetext{
${ }^{*}$ School of Economics, Fudan University. E-mail: jimmy.hing.chan@gmail.com

†'Department of Economics, NYU. E-mail: alessandro.lizzeri@nyu.edu

¥School of Economics and Finance, University of Hong Kong. E-mail: wsuen@econ.hku.hk

§Division of the Humanities and Social Sciences, Caltech. E-mail: lyariv@hss.caltech.edu

"This paper is the result of a merger of "Does Majority Rule Produce Hasty Decisions?" by Chan and Suen, and "Sequential Deliberation" by Lizzeri and Yariv. Ben Chiao, Jacques Cremer, John Morgan, Nicola Persico, Debraj Ray, Nikita Roketskiy, and Erik Snowberg provided very helpful conversations and feedback. We thank the editor, Marco Ottaviani, and six anonymous referees for many useful comments and suggestions. We gratefully acknowledge financial support from the Gordon and Betty Moore Foundation (grant 1158), the National Natural Science Foundation of China (project no. 71171125), the National Science Foundation (SES 0551014), the Shanghai Dongfang Xuezhe Program, and the Research Grants Council of Hong Kong (project no. HKU753911).
} 


\section{Introduction}

Many committees spend time deliberating issues and gathering information before reaching a decision or issuing a recommendation. Examples of such committees include juries, boards of directors, standard-setting organizations, congressional and university committees, and government agencies such as the FDA or the EPA. This paper presents a simple model of deliberation capturing some key features of committee decision processes.

We consider an environment in which committee members jointly decide, over time, how much information to collect before making one of two possible decisions (hiring or not hiring a candidate, adopting a new standard or sticking to the status quo, convicting or acquitting a defendant). Information arrives continuously (and publicly) according to a Weiner process. We allow agents' preferences to be heterogeneous in two dimensions: the urgency to reach a decision, i.e., agents' discount factors, and the appropriate standard of evidence to apply in order to adopt one of the two decisions, namely, agents' static preferences over the two alternatives. The case of a homogeneous committee reduces to the classic sequential sampling problem that has been studied in the statistics literature since Wald $(1947 a, b)$. In this approach, an agent acquires information sequentially. At each stage, the agent chooses whether to stop and take a decision, or to proceed to acquire additional information, which is costly. The optimal procedure involves a sequential likelihood ratio test, whereby intermediate values of the likelihood ratio require obtaining a new sample, while high (low) values of the likelihood ratio require stopping and taking one (or the other) decision.

The starting point of our analysis (Proposition 1) is a derivation of an analogue to the sequential sampling results for heterogeneous committees. We show that equilibria are still characterized in terms of waiting thresholds. The committee obtains additional information for intermediate values of the likelihood ratio, and takes decisions for high (low) values of the likelihood ratio. This characterization allows us to compare institutions (namely, deliberation protocols and voting rules). It also allows us to study the effects of a committee's composition, in terms of its members' discount factors and static preferences-on the length of deliberation, the accuracy of decisions, and the generated welfare for the committee and for society at large.

In evaluating voting rules, we need to balance several considerations. We show (in Proposition 2) that a weakness of majority rule is that deliberation can be excessively influenced by impatient agents. Since impatient agents prefer quicker decisions, they can be easily persuaded to change their votes. Under majority rule, it takes the change of only one vote to alter the outcome. Therefore, in equilibrium, patient agents supporting different alternatives cut short deliberations to capture any impatient member before she changes sides. Because quick decisions are strategic complements-if the supporters of one alternative expect the supporters of the other alternative to decide quickly, they will decide quickly as well-the mere presence of one impatient agent can cause the whole group to 
rush to a decision, even when all other agents are very patient. We call this phenomenon a hasty equilibrium. This insight crucially depends on allowing for two dimensions of heterogeneity. Indeed, in one-dimensional static voting environments, no agent with extreme preferences can influence the outcome under majority rule.

Super-majority rules, by comparison, are more robust against hasty decisions. Under super-majority rules, more than one vote must change to alter the outcome. Deliberations do not collapse unless the number of impatient agents is sufficiently large (Proposition 3). Moreover, because slow decisions are strategic substitutes, one very patient agent would not cause the group to deliberate indefinitely unless unanimity is required. Compared to majority rule, super-majority rules also tend to produce more accurate decisions and longer deliberations (Proposition 4). Hence, even when the agents themselves, who bear more of the delay costs, prefer majority rule, society at large, which benefits from a more accurate decision, may be better off with a super-majority rule.

An advantage of majority rule is that it always produces an outcome that is favored by a majority. Indeed, under majority rule, if a majority of agents prefers a different alternative to the one adopted in equilibrium, it can simply adopt the one preferred. Another advantage of majority rule is that all agents of the committee prefer it to stricter majority requirements in symmetric environments where all agents are equally patient and the static preferences for the two alternatives are balanced. Suppose that such a committee is comprised of an odd number of individuals. Majority rule then generates outcomes that would emerge from delegating all decisions to the most moderate member, with median static preferences. Therefore, in such environments members of a committee would unanimously prefer to delegate deliberation power to a moderate chairman rather than be governed by a deliberation rule such as unanimity (Proposition 5).

Super-majority rules have an important drawback. In an asymmetric environment, a high majority requirement may allow a small group with extreme preferences to hold out and force other members to accept an alternative that they do not like. This problem is the most severe under unanimity rule. In that case, the deliberation outcome may be preferred by only one member; the rest of group may all prefer the other alternative but vote for the inferior one to avoid costly delay (Proposition 6). Thus, while our results support the use of super-majority rules in situations where agents have diverse time preferences, they also warn against setting a very high majority requirement. In particular, requiring unanimity does not always build consensus.

One way to combine some features of majority and super-majority rules is to have a two-stage decision process. First, agents vote to end deliberation in a sequential process similar to that of our benchmark setup. When deliberation comes to a halt, agents vote simultaneously to select an alternative. We model the deliberation process with a threshold rule $k_{D}$ such that deliberation ends as soon as $k_{D}$ members of the committee vote to end deliberation. Decision rules are analogously captured by a rule $k_{d}$ that describes the 
specific qualified majority required for reaching a decision after deliberation stops. We show that in certain cases, decision rules are irrelevant, while deliberation rules always affect the length of deliberation and accuracy of ultimate decisions (Proposition 8). This separation, however, makes it impossible to have outcomes where agents vote against their static preferences in order to avoid costly delays (Proposition 7).

Our results are relevant to the design of deliberative mechanisms and for understanding the dynamics of information collection in a variety of collective decision processes such as R\&D, hiring decisions, FDA drug approval, and so on. In Section 8 we discuss in more detail two applications: standard-setting organizations along with juries. Standardsetting processes fit our benchmark setup in which deliberation and decision making are intertwined-decisions are sequential and are often of the form of "continue" or "take a decision" (approve or not). The case of juries fits our two-stage deliberation setting. Juries are an interesting application for our model for three main reasons. First, in juries, the deliberation process is clear-cut and circumscribed: there is a well-defined beginning and end of deliberation, the time it takes the jury to deliberate is measurable, and one single verdict is the typical outcome of such deliberation. Second, the jury setting allows us to contrast our analysis with much of the extant body of literature on deliberation that has focused predominantly on the jury context. Third, the empirical literature has documented some patterns of deliberation in juries that can be explained with our model.

\section{Related Literature}

In economics, the past two decades have delivered a rich collection of work on committee decision making; see Li and Suen (2009) for an extended survey. Our paper ties directly to several sets of studies.

There is a literature on committee decision making that focuses on how private information of individual agents is aggregated under alternative voting rules. A key finding in this literature is that unanimity leads to less informative outcomes than majority rule. ${ }^{1}$ We identify additional disadvantages of majority rule, but our result that unanimity can lead to more accurate outcomes than majority rules contrasts with these results.

Our paper is also related to recent contributions that adopt a collective search approach (Albrecht, Anderson, and Vroman 2010; Compte and Jehiel 2010; 2011; Moldovanu and Shi 2013). Of these, the most related is the paper of Albrecht, Anderson, and Vroman (2010). They find that in a heterogeneous search committee, each committee member will apply a lower acceptance standard than what she would have were she the sole decision

\footnotetext{
${ }^{1}$ See Feddersen and Pesendorfer (1998). Persico (2004) also obtains this result when allowing for private information collection prior to voting. Austen-Smith and Feddersen (2006) consider a round of cheap-talk communication before voting and also show that unanimity leads to less communication and poorer information aggregation. Gerardi and Yariv $(2007,2008)$ depart from these papers by studying general communication protocols. They show that the set of equilibrium outcomes is invariant to the voting rules, as long as they are non-unanimous. In fact, unanimous voting rules generate a subset of equilibrium outcomes.
} 
maker. This effect is also present in our model. However, in their model, in contrast with ours, having one committee member who is willing to accept proposals very quickly cannot cause the rest of the committee to stop searching entirely. The key difference is that, in the case of search, independent draws of alternatives appear every period, and the group's decision is whether to adopt that alternative or to continue with search. In our setup, alternatives are fixed but new information about the existing alternatives appears over time, and the group's decision is when to stop collecting evidence and decide which alternative to adopt. The relative merits of different alternatives evolve as information arrives: the leading alternative is expected to still lead within a short interval of time, but has a possibility of being overtaken by another alternative after sufficient information has been accumulated over a longer interval. ${ }^{2}$

In our model a majority may decide to act early to prevent members from changing side. A similar result is obtained by Strulovici (2010) in the context of dynamic collective experimentation. In his model a new policy may affect different voters differently. He shows that, in equilibrium, a group that is pessimistic about the new policy may vote to end the experiment prematurely to prevent the new policy from gaining more supporters.

From a technical perspective, the starting point of our analysis is Wald (1947a, 1947b), who pioneered the study of sequential testing, and provided a characterization of the optimal test as a sequential likelihood ratio test. Dvoretsky, Keifer, and Wolfowitz (1953), Mikhalevich (1958), and Shiryaev (1967) gave an early treatment of the hypothesis testing problem in continuous time. ${ }^{3}$ Two recent papers also apply the framework of sequential testing. Henry and Ottaviani (2014) study of the approval process when a firm conducting clinical trials needs approval from a regulator such as the FDA. They allow for the possibility that the firm may misrepresent the evidence and show that it may not be optimal to forbid such misrepresentation. Gul and Pesendorfer (2012) study the competition between two political parties to provide public information that may influence voters' choices. In equilibrium, each party chooses a threshold and stops providing information once the voter's belief is less favorable than that threshold. This is similar to our model under unanimity rule. However, in their model, since the parties have opposite

\footnotetext{
${ }^{2}$ Messner and Polborn (2012) study a two-period model where voters receive information over time about the desirability of an irreversible decision. The main message of that paper is that the optimal voting rule requires a super-majority. Bognar, Meyer-ter-Vehn, and Smith (2015) also study a model of dynamic deliberation, but with very different ingredients. In their model, jurors have private information about a payoff-relevant state. They assume that jurors sequentially exchange coarse messages. That model produces many equilibria that can be ranked in terms of generated welfare. When there is no discounting, they show that longer conversations are better. A related paper is that of Eso and Fong (2008), who study a dynamic cheap talk model with multiple senders, where the receiver can choose when to make her decision. They show that when the senders are all informed of the state of nature, a perfect Bayesian equilibrium exists with instantaneous, full revelation, regardless of the size and direction of the senders' biases. Wilson (2014) considers exogenous costs for both sending messages and receiving them, and illustrates the dependence of effective communication on agents' quality of information and messaging costs.

${ }^{3}$ See also De Groot (1970) for a modern exposition, and Moscarini and Smith (2001) for an extension that allows for richer sampling strategies.
} 
policy preference, their choices are always strategic substitutes-if one party stops later, the other party will stop earlier. In contrast, in our model, because the voters share a common interest conditional on the true state, their one-sided best-response functions are non-monotone.

\section{The Model}

A group of $2 m-1$ agents chooses between two alternatives, $\alpha$ and $\beta .^{4}$ The payoff from each alternative depends on the underlying state $\omega \in\{A, B\}$. In state $A$, agent $i$ 's payoff from $\alpha$ is 1 , and his payoff from $\beta$ is 0 . In state $B$, agent $i$ 's payoff from $\alpha$ is 0 , and his payoff from $\beta$ is $e^{v_{i}}$. Thus, all agents prefer action $\alpha$ in state $A$ and action $\beta$ in state $B$, but agents differ in the intensity of this preference, their static preferences, and $v_{i} \in \mathbb{R}$ is a measure of the intensity of agent $i$ 's preference for $\beta$ relative to $\alpha$. Agents' static preferences are heterogenous, with $v_{1}<\ldots<v_{2 m-1}$. The median static preference is $v_{m}$. Agents do not observe the state but share a common prior belief. It is convenient to represent agents' beliefs by the log ratio of the probabilities of the two states. We let $\theta_{0}=\log (\operatorname{Pr}[\omega=A] / \operatorname{Pr}[\omega=B])$ represent the initial belief. With this parameterization, the probability of state $A$ is given by $e^{\theta} /\left(1+e^{\theta}\right)$ when the belief is $\theta$. The immediate expected payoff from choosing $\alpha$ is higher than that from choosing $\beta$ for agent $i$ if and only if $\theta \geq v_{i}$.

Time is continuous on $[0, \infty)$. At every instant $t$, each agent independently votes for $\alpha, \beta$, or neither. We initially focus on a simple class of decision rules. Under decision rule $k \in\{m, m+1, \ldots, 2 m-1\}$, an alternative is adopted at time $t$ if it receives $k$ votes or more. Voting continues until either $\alpha$ or $\beta$ receive sufficient votes. Voting for neither alternative is effectively a vote in favor of continuing to gather information. Voting for one alternative is also a vote in favor of stopping information acquisition. ${ }^{5}$ We call rule $k=m$ majority rule, $k>m$ super-majority rule, and $k=2 m-1$ unanimity rule. The usage is natural in contexts where alternatives are treated symmetrically, and deliberation continues until an alternative has received enough support. In Section 7 we consider an alternative structure by modeling a two-stage deliberation process that separates voting on information gathering from voting on the final decision.

Each agent $i$ discounts the future at a rate $r_{i}$. If an alternative is chosen at time $t$, then agent $i$ 's payoff is discounted by the factor $e^{-r_{i} t}$. In general, agents are heterogeneous both in their static preferences $v_{i}$ and in their time preferences $r_{i}{ }^{6}$

\footnotetext{
${ }^{4}$ The assumption of an odd number of agents is used only when we consider majority rule.

${ }^{5}$ Throughout the paper, majority rule or super-majority rule refer to an "absolute" rule. Under an absolute rule, abstention is treated as a vote to support neither alternative. In contrast, a "simple" rule discards abstention votes in the tally. This distinction is based on Riker (1982). Our results would not change under the following alternative rule: each agent can choose to support $\alpha$, support $\beta$, delay, or abstain; an alternative is adopted if the fraction of votes, excluding abstentions, passes a threshold. Because there is no private information in this model, there is no strategic advantage to abstention relative to voting for delay (Feddersen and Pesendorfer 1996).

${ }^{6}$ While our setup uses heterogeneous discount rates to model different preferences over how quickly to
} 
Public information arrives continuously as long as deliberation has not stopped and an alternative has been chosen. The arrival of information is represented by a Wiener process $\mathrm{d} S$ that has a positive drift $\mu$ and an instantaneous variance $\rho^{2}$ if the state is $A$, or drift $-\mu$ and variance $\rho^{2}$ if the state is $B$. For any time $t>0$, the accumulated evidence $S_{t}$ is a sufficient statistic for all the information that has arrived before $t$. The log-likelihood ratio of observing $S_{t}=s$ under the two states is

$$
\log \frac{h((s-\mu) / \rho)}{h((s+\mu) / \rho)}=\frac{2 \mu s}{\rho^{2}},
$$

where $h(\cdot)$ is the standard normal density function. Hence, a higher observed value of the accumulated evidence $S_{t}$ is stronger evidence in favor of state $A$. The log posterior probability ratio is given by the sum of the log prior probability ratio and the log-likelihood ratio. So, if we let $S_{t}^{\prime}=2 \mu S_{t} / \rho^{2}$, the common belief of the group at time $t$ is given by:

$$
\theta_{t}=\theta_{0}+S_{t}^{\prime}
$$

Denote $\mu^{\prime} \equiv 2 \mu^{2} / \rho^{2}$. Then, agents' common belief is given by a Wiener process, with drift $\mu^{\prime}$ and instantaneous variance $2 \mu^{\prime}$ under state $A$, and drift $-\mu^{\prime}$ and variance $2 \mu^{\prime}$ under state $B$. A higher value of $\mu^{\prime}$ (higher $\mu$ or lower $\rho$ ) indicates a more informative deliberative process.

When $m=1$, decisions correspond to an individual. This is the classic case analyzed in the literature on sequential analysis that started with Wald $(1947 a, b) .{ }^{7}$ In this case, predictions are unique and can be characterized as follows.

Proposition 0. When agent $i$ is the only agent in the group (i.e., $m=1$ ), a unique equilibrium exists and is characterized by two thresholds $g_{i}^{*}<G_{i}^{*}$ such that:

- the agent stops information collection and chooses $\alpha$ whenever $\theta_{t} \geq G_{i}^{*}$;

- the agent stops information collection and chooses $\beta$ whenever $\theta_{t}<g_{i}^{*}$;

- the agent continues collecting information whenever $\theta_{t} \in\left[g_{i}^{*}, G_{i}^{*}\right)$.

When $m>1$, in principle, an agent's decision at time $t$ could be a function of the entire sample path of $S_{t}$, her own decisions, and other agents' decisions before time $t$. However, the difference between the expected payoffs of the two decisions $\alpha$ and $\beta$ depends solely

act, a very similar model can be constructed with explicit information acquisition costs. Suppose that, for agent $i$ the cost of information collection is $\delta \mathrm{d} t$ for a time interval of length $\mathrm{d} t$, and the payoffs from $\alpha$ and $\beta$ are $\lambda_{i}$ and $\lambda_{i} e^{v_{i}}$, respectively. Agents with low values of $\lambda_{i}$ are low-stake voters: their primary concern is to reduce the explicit information collection cost. In such an alternative setup, low-stake agents play a similar role to that played by impatient agents in our model.

${ }^{7}$ Wald studied a discrete time process. The continuous time case was studied by Dvoretsky et al. (1953). See also Mikhalevich (1958) and Shiryaev (1967). 
on the current belief $\theta_{t}$ and is strictly increasing in it. We focus on equilibria in which agents adopt Markov cutoff strategies (much like the optimal policies characterized by Wald for an individual decision maker). Formally, agent $i$ 's strategy $\sigma_{i}$ is represented by a pair of cutoffs $\left(g_{i}, G_{i}\right)$, with $g_{i} \leq G_{i}$. Strategy $\left(g_{i}, G_{i}\right)$ means voting for $\alpha$ when $\theta_{t} \geq G_{i}$, voting for $\beta$ when $\theta_{t}<g_{i}$, and abstaining when $\theta_{t} \in\left[g_{i}, G_{i}\right) .^{8}$

For any strategy profile $\sigma=\left(\sigma_{1}, \ldots, \sigma_{2 m-1}\right)$, let $G^{[k]}(\sigma)$ denote the $k$-th smallest $G_{i}$ in $\sigma$, and let $g^{[k]}(\sigma)$ denote the $k$-th largest $g_{i}$ in $\sigma$. We call the interval $\left(g^{[k]}(\sigma), G^{[k]}(\sigma)\right)$ the waiting region, because the group does not make a decision as long as the belief stays within this interval. The width of the waiting region, $G^{[k]}(\sigma)-g^{[k]}(\sigma)$, is an indicator of the expected time to make a decision. Note, however, that a wider waiting region does not necessarily mean that the group always waits longer, unless two waiting regions are nested (i.e., one waiting region contains the other). If two waiting regions are nested, decisions reached with the larger waiting region are more accurate because each alternative would be adopted when the probability of its corresponding state is higher.

Let $u_{i}(g, G \mid \theta)$ represent the payoff to agent $i$ when the belief is $\theta$ and the waiting region is $(g, G)$. If $\theta \geq G$, then $\alpha$ is adopted and the expected payoff is

$$
u_{i}(g, G \mid \theta)=\frac{e^{\theta}}{1+e^{\theta}} .
$$

If $\theta<g$, then $\beta$ is adopted and the expected payoff is

$$
u_{i}(g, G \mid \theta)=\frac{e^{v_{i}}}{1+e^{\theta}} .
$$

For $\theta \in[g, G)$, the payoff function satisfies

$$
u_{i}(g, G \mid \theta)=e^{-r_{i} \mathrm{~d} t} \mathbb{E}\left[u_{i}\left(g, G \mid \theta+\mathrm{d} S^{\prime}\right)\right] .
$$

Because $\mathrm{d} S^{\prime}$ is a diffusion process with expected drift $\mu^{\prime}\left(e^{\theta}-1\right) /\left(1+e^{\theta}\right)$ and instantaneous variance $2 \mu^{\prime}$, we can use Ito's lemma to derive a differential equation in $u_{i}$. Solving this differential equation and imposing the value-matching condition at the two boundaries of the waiting region, we obtain:

$$
u_{i}(g, G \mid \theta)=\frac{e^{\theta}}{1+e^{\theta}} \Psi_{i}(g, G \mid \theta)+\frac{e^{v_{i}}}{1+e^{\theta}} \psi_{i}(g, G \mid \theta),
$$

\footnotetext{
${ }^{8}$ Of course, at a formal level, our analysis can be thought of as static: Individuals can commit to thresholds, and then go through the voting process.
} 
where

$$
\begin{aligned}
& \Psi_{i}(g, G \mid \theta)=\frac{e^{-R_{1}(\theta-g)}-e^{-R_{2}(\theta-g)}}{e^{-R_{1}(G-g)}-e^{-R_{2}(G-g)},} \\
& \psi_{i}(g, G \mid \theta)=\frac{e^{-R_{1}(G-\theta)}-e^{-R_{2}(G-\theta)}}{e^{-R_{1}(G-g)}-e^{-R_{2}(G-g)}},
\end{aligned}
$$

with

$$
R_{1}=\frac{1}{2}\left(1-\sqrt{1+\frac{4 r_{i}}{\mu^{\prime}}}\right), \quad R_{2}=\frac{1}{2}\left(1+\sqrt{1+\frac{4 r_{i}}{\mu^{\prime}}}\right) .
$$

Note that $R_{2}>1, R_{1}<0$, and $R_{1}+R_{2}=1$. The values of $R_{1}$ and $R_{2}$ depend on $i$, but we omit this dependence in the notation to avoid clutter. ${ }^{9}$

The two functions $\Psi_{i}$ and $\psi_{i}$ can be given a statistical interpretation (Cox and Miller 1965). When $\theta \in(g, G)$, agent $i$ 's payoff depends on which threshold is reached first and on the length of time it takes to reach it. The function $\Psi_{i}(g, G \mid \theta)$ provides the probability of adopting $\alpha$ in state $A$ when the current belief is $\theta$, times the expected discount factor conditional on the belief reaching the threshold $G$ before reaching $g$ in state $A$. The function $\psi_{i}$ can be interpreted analogously.

Definition 1. Let $(\hat{g}, \hat{G})=\left(g^{[k]}(\sigma), G^{[k]}(\sigma)\right)$. A strategy profile $\sigma$ is an equilibrium under decision rule $k$ if the following conditions are satisfied for any agent $i$ :

1. For any belief $\theta$ and any strategy $\sigma_{i}^{\prime}$

$$
u_{i}(\hat{g}, \hat{G} \mid \theta) \geq u_{i}\left(g^{[k]}\left(\sigma_{i}^{\prime}, \sigma_{-i}\right), G^{[k]}\left(\sigma_{i}^{\prime}, \sigma_{-i}\right) \mid \theta\right) .
$$

2. (a) $G_{i}>(<) \hat{G}$ if $\partial u_{i} /\left.\partial G\right|_{(\hat{g}, \hat{G} ; \theta=\hat{G})}>(<) 0$;

(b) $g_{i}>(<) \hat{g}$ if $\partial u_{i} /\left.\partial g\right|_{(\hat{g}, \hat{G} ; \theta=\hat{g})}>(<) 0$.

Condition 1 of Definition 1 is the standard Nash equilibrium requirement. Condition 2 says that agent $i$ does not vote for an alternative at the threshold if her marginal gain from extending the threshold is positive, and, conversely, that she votes for an alternative before the belief reaches the threshold for that alternative if her marginal gain from reducing the threshold is positive. Note that as long as the decision rule $k$ is not the unanimity rule, for any pair of thresholds $(g, G)$, it would be a Nash equilibrium in our model for all agents to adopt the same strategy $(g, G)$, because no agent could unilaterally change the waiting region. Condition 2 rules out trivial equilibria that commonly arise in voting games because of the use of weakly dominated strategies, e.g. ones involving all agents voting unanimously for one alternative regardless of beliefs.

\footnotetext{
${ }^{9}$ Note that $R_{1}$ and $R_{2}$ are homogeneous of degree 0 in $r_{i} / \mu^{\prime}$. In principle, we could therefore carry the analysis using discount factors normalized by $\mu^{\prime}$. We maintain our notation for transparency.
} 


\section{Best Responses in Stopping Decisions}

This section covers some of the basic features of best responses in our setting. The results underlie our equilibrium characterization in later sections. While the analysis here constitutes an important preliminary step, some of which may be of independent interest, it is more technical in nature. A reader interested only in the qualitative insights of our analysis may skip this section.

In the classic individual optimal-stopping problem, an agent $i$ chooses $\left(g_{i}, G_{i}\right)$ to maximize $u_{i}(g, G \mid \theta)$. The group decision model differs in that the influence of each agent is constrained by the cutoffs of other agents. Indeed, in terms of adopting $\alpha$, there are three possible cases to consider for agent $i$ : (i) The posterior belief exceeds $G^{[k]}\left(\sigma_{-i}\right)$. In this case, there is a $k$-majority of other agents favoring the adoption of $\alpha$ immediately and agent $i$ has no impact on the decision. (ii) The posterior belief is lower than $G^{[k-1]}\left(\sigma_{-i}\right)$, so that fewer than $k-1$ other agents agree to adopt $\alpha$. In this case, the agent cannot force the adoption of $\alpha$. (iii) The posterior belief is between $G^{[k-1]}\left(\sigma_{-i}\right)$ and $G^{[k]}\left(\sigma_{-i}\right)$. That is, exactly $k-1$ other agents want to adopt $\alpha$ immediately. In this case agent $i$ can affect the stopping decision, i.e., she may be pivotal. Heterogeneity implies that agents disagree on their ideal thresholds for stopping deliberation, and therefore, the identity of the pivotal agent depends on the posterior. From the perspective of the agent, her problem is a constrained version of the single-person problem, where she takes as given the fact that there is a region where she cannot stop information collection.

We say that agent $i$ 's span of control over the upper threshold is the interval

$$
I^{G}\left(\sigma_{-i}\right)=\left[G^{[k-1]}\left(\sigma_{-i}\right), G^{[k]}\left(\sigma_{-i}\right)\right] .
$$

Analogously, we define $I^{g}\left(\sigma_{-i}\right)=\left[g^{[k]}\left(\sigma_{-i}\right), g^{[k-1]}\left(\sigma_{-i}\right)\right]$ as agent $i^{\prime}$ s span of control over the lower threshold. Then, Condition 1 of the equilibrium definition is equivalent to requiring that $\left(g^{[k]}(\sigma), G^{[k]}(\sigma)\right)$ be a solution to the following constrained maximization problem for each agent $i$ and for every belief $\theta$ :

$$
\max _{g, G} u_{i}(g, G \mid \theta) \quad \text { subject to }(g, G) \in I^{g}\left(\sigma_{-i}\right) \times I^{G}\left(\sigma_{-i}\right) .
$$

We proceed as follows. We first study a one-sided stopping problem in which an agent takes one of the stopping boundaries as fixed and chooses the other stopping boundary. We show that the corresponding best responses are well behaved. We then use the solution to this one-sided problem to characterize the solution to the constrained two-sided stopping problem (1). Intuitively, when contemplating voting in favor of, say, the $\alpha$ alternative, a committee member has to consider the circumstances under which the $\beta$ alternative will be selected, which are determined in equilibrium. In that respect, the committee member uses a one-sided best response, subject to the constraints imposed by her span of 
control.

We first describe two important consequences of a change in one stopping boundary on an agent's utility, holding the other stopping boundary fixed:

Lemma 1. For $\theta \in(g, G)$, the following properties hold for any agent $i$ :

1. Dynamic consistency: The signs of $\partial u_{i} / \partial G$ and $\partial u_{i} / \partial g$ do not depend on $\theta$.

2. Single crossing: (a) If $\partial u_{i} / \partial G \geq 0$ at $G^{\prime}>G$, then $\partial u_{i} / \partial G>0$ at $G$ (holding $g$ constant). (b) If $\partial u_{i} / \partial g \leq 0$ at $g^{\prime}<g$, then $\partial u_{i} / \partial g<0$ at $g$ (holding $G$ constant).

The partial derivative of $u_{i}$ with respect to a threshold reflects the trade-off between the cost of delay and the value of additional information. Part 1 of Lemma 1 says that this trade-off is dynamically consistent: if an agent prefers to extend a threshold when her belief reaches it, she prefers to extend this threshold before reaching it. Part 2 of Lemma 1 says that $u_{i}$ is single-peaked in each threshold, holding the other constant. Intuitively, if an agent prefers waiting to selecting $\alpha$ at a later threshold $G^{\prime}$, she strictly prefers waiting to selecting $\alpha$ at an earlier threshold $G$, where the case for $\alpha$ is weaker.

We can now turn to the characterization of one-sided best responses. For any agent $i$, denote by $\phi_{i}(G)$ the lower best-response function for agent $i$, which gives, for any fixed $G$, the optimal lower cutoff $g$ that maximizes $u_{i}(g, G \mid \theta)$ subject to $g \leq G$. The dynamic consistency and single-crossing properties of Lemma 1 guarantee that $\phi_{i}(G)$ is well defined and is independent of the current belief $\theta$. Similarly, the upper best-response function $\Phi_{i}(g)$ is defined as the optimal upper cutoff $G$ that maximizes $u_{i}(g, G \mid \theta)$ subject to $G \geq g$. Recall that $g_{i}^{*}$ and $G_{i}^{*}$ are the optimal cutoffs for agent $i$ when she can choose both thresholds. The point $\left(g_{i}^{*}, G_{i}^{*}\right)$ must be a fixed point of $\left(\phi_{i}, \Phi_{i}\right)$.

Lemma 2. The following properties hold for any agent $i$ :

1. (a) $\Phi_{i}(g)$ is continuous everywhere and is twice differentiable except at $g=v_{i}$, with $\Phi_{i}(g)>$ $g$ if $g<v_{i}$ and $\Phi_{i}(g)=g$ otherwise.

(b) $\phi_{i}(G)$ is continuous everywhere and is twice differentiable except at $G=v_{i}$, with $\phi_{i}(G)<G$ if $G>v_{i}$ and $\phi_{i}(G)=G$ otherwise.

2. (a) For $G>v_{i}, \phi_{i}^{\prime \prime}(G)>0, \phi_{i}^{\prime}(G)<1$, and $\phi_{i}(G)$ reaches a minimum when $G=G_{i}^{*}$ and goes to $\infty$ as $G$ goes to $\infty$.

(b) For $g<v_{i}, \Phi_{i}^{\prime \prime}(g)<0, \Phi_{i}^{\prime}(g)<1$, and $\Phi_{i}(g)$ reaches a maximum when $g=g_{i}^{*}$ and goes to $-\infty$ as g goes to $-\infty$.

3. (a) For any $G, \phi_{i}(G)$ increases in $v_{i}$ and increases in $r_{i}$, with $\lim _{v_{i} \rightarrow-\infty} \phi_{i}(G)=-\infty$ and $\lim _{r_{i} \rightarrow \infty} G-\phi_{i}(G)=0$.

(b) For any $g, \Phi_{i}(g)$ increases in $v_{i}$ and decreases in $r_{i}$, with $\lim _{v_{i} \rightarrow \infty} \Phi_{i}(g)=\infty$ and $\lim _{r_{i} \rightarrow \infty} \Phi_{i}(g)-g=0$. 


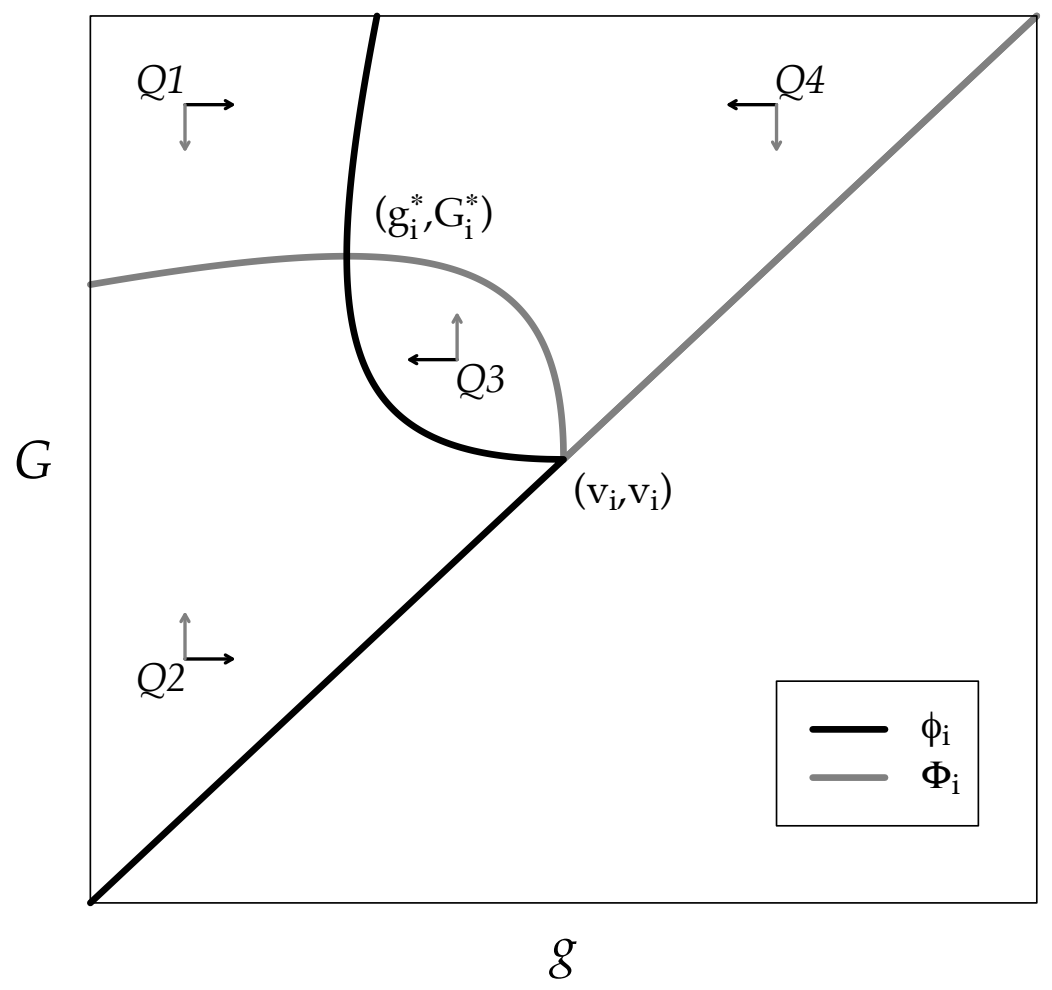

Figure 1. The upper best-response function $\Phi_{i}(g)$ is increasing for $g<g_{i}^{*}$, decreasing for $g \in\left(g_{i}^{*}, v_{i}\right)$, and coincides with the 45-degree line for $g>v_{i}$. The two best-response functions partition the space into four regions. The arrows associated with each region show that agent $i$ 's utility increases if the cutoff moves in the indicated direction. This figure is drawn using the parameters $v_{i}=0, r_{i}=0.039$, and $\mu^{\prime}=0.1$.

Figure 1 depicts a typical pair of lower and upper best-response functions $\phi_{i}$ and $\Phi_{i}$. We focus on the properties of $\phi_{i}$ in the following discussion; the properties of $\Phi_{i}$ are analogous.

If the threshold $G$ for adopting $\alpha$ is set below $v_{i}$, there is no point in delaying the adoption of $\beta$, as agent $i$ would still prefer $\beta$ to $\alpha$ when her belief reaches the threshold for adopting $\alpha$. Thus, $\phi_{i}(G)=G$. By contrast, if $G>v_{i}$, agent $i$ strictly prefers $\alpha$ to $\beta$ when her belief is at $G$. Hence, when her belief is sufficiently close to $G$, even an extremely impatient agent $i$ will prefer delaying the decision $\beta$, which, at this belief is the "wrong" decision for her, in the hope that the belief will reach $G$ and $\alpha$ will be chosen instead. Thus, $\phi_{i}(G)<G$. Note that $\phi_{i}(G)$ is continuous at $G=v_{i}$. When $G$ is just above $v_{i}$, the difference in expected payoff between $\alpha$ and $\beta$ is very small at $G$. Hence, agent $i$ gains little from delaying the adoption of $\beta$.

Part 2 of Lemma 2 describes agent $i$ 's best response when $G>v_{i}$. Some of these properties are technical, but two features are of economic interest. First is the non-monotonicity 
of $\phi_{i}(G)$. Intuitively, $\alpha$ is chosen "too early" when $G<G_{i}^{*}$ (the unconstrained optimal cutoff), and is chosen "too late" when $G>G_{i}^{*}$. In either case, the agent responds by adopting $\beta$ earlier. The possibility that a loss of control (over the upper boundary) may reduce the incentive to obtain information is discussed in Albrecht, Anderson, and Vroman (2010) in the context of a collective search model, and by Strulovici (2010) in a two-armed-bandit experiment setting. The non-monotonicity of $\phi_{i}$ implies there is a strategic difference between too much and too little waiting. Quick decisions are strategic complements; slow decisions are strategic substitutes. In other words, excessive deliberation is self-limiting, while rushing to a decision is self-reinforcing.

The second feature of economic interest is that $\phi_{i}(G)$ is unbounded from above. In particular, it is possible that $\phi_{i}(G)>v_{i}$ when $G$ is sufficiently large. An agent who prefers $\alpha$ to $\beta$ may nevertheless vote to adopt $\beta$ immediately if she anticipates that she would have to wait for a very long time for the group to adopt $\alpha$.

Part 3 of Lemma 2 describes how $\phi_{i}$ varies with $v_{i}$ and $r_{i}$. In terms of Figure 1 , an increase in $v_{i}$ would shift both $\phi_{i}$ and $\Phi_{i}$ in the north-east direction, causing agent $i$ to adopt $\beta$ earlier and $\alpha$ later. An increase in $r_{i}$ "bends" $\phi_{i}$ and $\Phi_{i}$ toward the 45-degree line, which prompts agent $i$ to adopt both $\alpha$ and $\beta$ earlier. As agent $i$ becomes extremely impatient, the lower best-response threshold becomes arbitrarily close to the upper bestresponse threshold.

Let $I^{G}\left(\sigma_{-i}\right)=\left[\underline{G}_{i}, \bar{G}_{i}\right]$ and $I^{g}\left(\sigma_{-i}\right)=\left[\underline{g}_{i}, \bar{g}_{i}\right]$. Given the single-crossing property (Lemma $1), \hat{g}$ is optimal in $\left[\underline{g}_{i}, \bar{g}_{i}\right]$ for any belief $\theta$ given $\hat{G}$ only if it satisfies:

$$
\hat{g} \begin{cases}\geq \phi_{i}(\hat{G}) & \text { if } \hat{g}=\underline{g}_{i}, \\ =\phi_{i}(\hat{G}) & \text { if } \hat{g} \in\left(\underline{g}_{i}, \bar{g}_{i}\right), \\ \leq \phi_{i}(\hat{G}) & \text { if } \hat{g}=\bar{g}_{i} .\end{cases}
$$

Similarly, $\hat{G}$ is optimal in $\left[\underline{G}_{i}, \bar{G}_{i}\right]$ for any belief $\theta$ given $\hat{g}$ only if it satisfies:

$$
\hat{G} \begin{cases}\geq \Phi_{i}(\hat{g}) \quad \text { if } \hat{G}=\underline{G}_{i}, \\ =\Phi_{i}(\hat{g}) \quad \text { if } \hat{G} \in\left(\underline{G}_{i}, \bar{G}_{i}\right), \\ \leq \Phi_{i}(\hat{g}) \quad \text { if } \hat{G}=\bar{G}_{i} .\end{cases}
$$

The following lemma shows that these conditions are also sufficient; that is, they characterize Condition 1 of our equilibrium definition and therefore provide the connection between one-sided best responses and the equilibria of our model.

Lemma 3. A pair of thresholds $(\hat{g}, \hat{G})$, with $\hat{g}<\hat{G}$, solves the constrained optimization problem (1) for all beliefs $\theta$ if and only if it satisfies conditions (2) and (3).

Lemma 3 plays a crucial role in our analysis. Despite the fact that dynamic consistency 
holds for one-sided preferences, dynamic consistency does not generally hold when both thresholds change at the same time. An agent may prefer waiting region $(g, G)$ to $\left(g^{\prime}, G^{\prime}\right)$ at some belief $\theta$, but have the opposite preference at some other belief $\theta^{\prime}$. Nevertheless, Lemma 3 shows that a solution to the constrained optimization problem (1) for all $\theta$ exists. Furthermore, it reduces our original two-sided collective stopping problem, which is difficult to solve directly, into two readily solvable one-sided problems. In the proof of Lemma 3, we use part 2 of Lemma 2 to establish that only one pair of cutoffs in the constraint set satisfies both (2) and (3). Because (2) and (3) are necessary for constrained maximization, this unique cutoff pair must be optimal in the constraint set. ${ }^{10}$

\section{Equilibrium Analysis}

Given decision rule $k$, define the lower pivotal best-response function $\phi_{\text {piv }}(G ; k)$ to be the $k$-th largest $\phi_{i}(G)$ for each $G$. Thus, in a one-sided stopping problem, where $\alpha$ is adopted at $G$, there are $k$ agents who support $\beta$ when the belief reaches $\phi_{p i v}(G ; k)$. Similarly, define the upper pivotal best-response function $\Phi_{p i v}(g ; k)$ to be the $k$-th smallest $\Phi_{i}(g)$ for each $g$. Notice that these do not necessarily correspond to cutoffs any of the agents would use if they were deciding on their own. In what follows, we sometimes suppress the argument $k$ when doing so does not cause confusion. If the static preferences $v_{i}$ are all the same and agents only differ in their discount factors $r_{i}$, then every $k$ is associated with a unique pivotal agent for both thresholds: this is the $k$-th most impatient agent. If the discount factors $r_{i}$ are all the same and agents only differ in their static preferences $v_{i}$ then, as we will see, there are different pivotal agents for the two thresholds: these are the agents with the $k$-th lowest and the $k$-th highest static preferences. More generally, however, when agents are heterogeneous in both dimensions, determining the identity of the pivotal agents is not so simple. Furthermore, because individual best-response functions may cross if agents have different time preferences, the identity of the pivotal agent who supports $\beta$ may change as $G$ changes. See Figure 2 for an illustration.

The following proposition establishes some basic properties of equilibrium.

Proposition 1. For any decision rule $k$, any $(\hat{g}, \hat{G})$ is an equilibrium outcome of the deliberation game if and only if it is a fixed point of $\left(\phi_{\text {piv }}, \Phi_{\text {piv }}\right)$ and $\hat{g}<\hat{G}$. An equilibrium of the deliberation game exists and the equilibrium waiting region must be non-degenerate. When there are multiple equilibria, the equilibrium waiting regions are nested. Equilibrium is unique if the decision rule requires unanimity or if there is a single dimension of heterogeneity.

It is obvious that any equilibrium cutoffs must be a fixed point of the pivotal bestresponse functions. Sufficiency follows from Lemma 3: if $(\hat{g}, \hat{G})$ is an unequal fixed point

\footnotetext{
${ }^{10}$ As the utility function $u_{i}$ is not quasi-concave in cutoffs, the Kuhn-Tucker conditions in general are not sufficient for optimality in an arbitrary convex constraint set.
} 


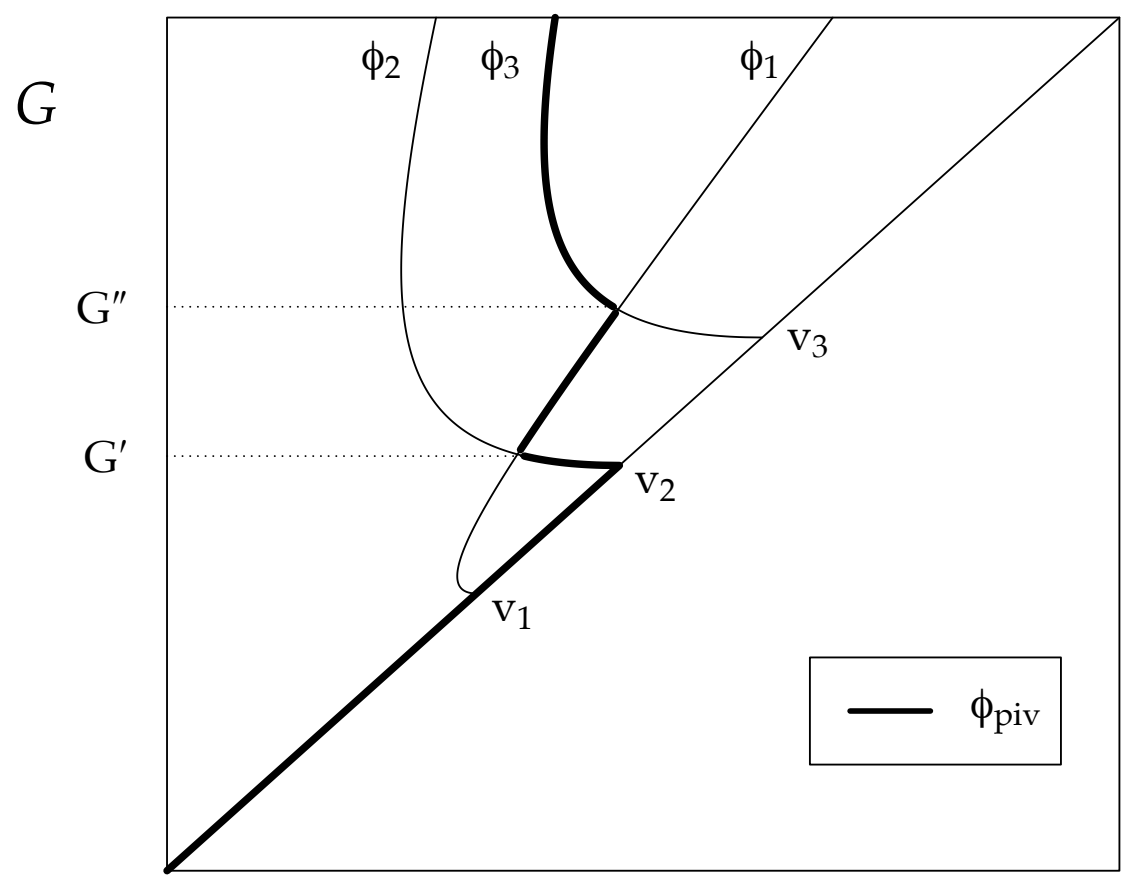

$g$

Figure 2. The bold line shows the lower pivotal best-response function when there are three agents and when the decision rule is majority rule. For $G<v_{2}, \phi_{\text {piv }}$ coincides with the 45-degree line. For fixed $G$, the median agent (agent 2) is pivotal in the decision to adopt $\beta$ if $G \in\left(v_{2}, G^{\prime}\right)$. Agent 1 is more impatient than others; he would prefer to adopt $\beta$ earlier than agent 2 (but later than agent 3 ) if $G \in\left(G^{\prime}, G^{\prime \prime}\right)$. For $G>G^{\prime \prime}$, agent 1 prefers to adopt $\beta$ the earliest and agent 3 becomes pivotal. In this figure, $\left(v_{1}, v_{2}, v_{3}\right)=$ $(-0.6,0,0.6),\left(r_{1}, r_{2}, r_{3}\right)=(0.6,0.039,0.039)$, and $\mu^{\prime}=0.1$.

of $\left(\phi_{\text {piv }}, \Phi_{\text {piv }}\right)$, i.e., $\hat{g}<\hat{G}$, then the strategy profile $\sigma$ where, for each $i$,

$$
\sigma_{i}=\left(\phi_{i}(\hat{G}), \Phi_{i}(\hat{g})\right)
$$

satisfies (2) and (3). Under majority rule, $\left(v_{m}, v_{m}\right)$ is a fixed point of $\left(\phi_{\text {piv }}, \Phi_{\text {piv }}\right)$ but not an equilibrium, because agent $m$ can gain by waiting a little before adopting one of the alternatives.

Multiplicity of Equilibria. Multiple equilibria can arise for non-unanimous decision rules when there is heterogeneity in both dimensions. Figure 3 provides an example. Thus, expectations can play an important role in deliberations. Agents may decide slowly 


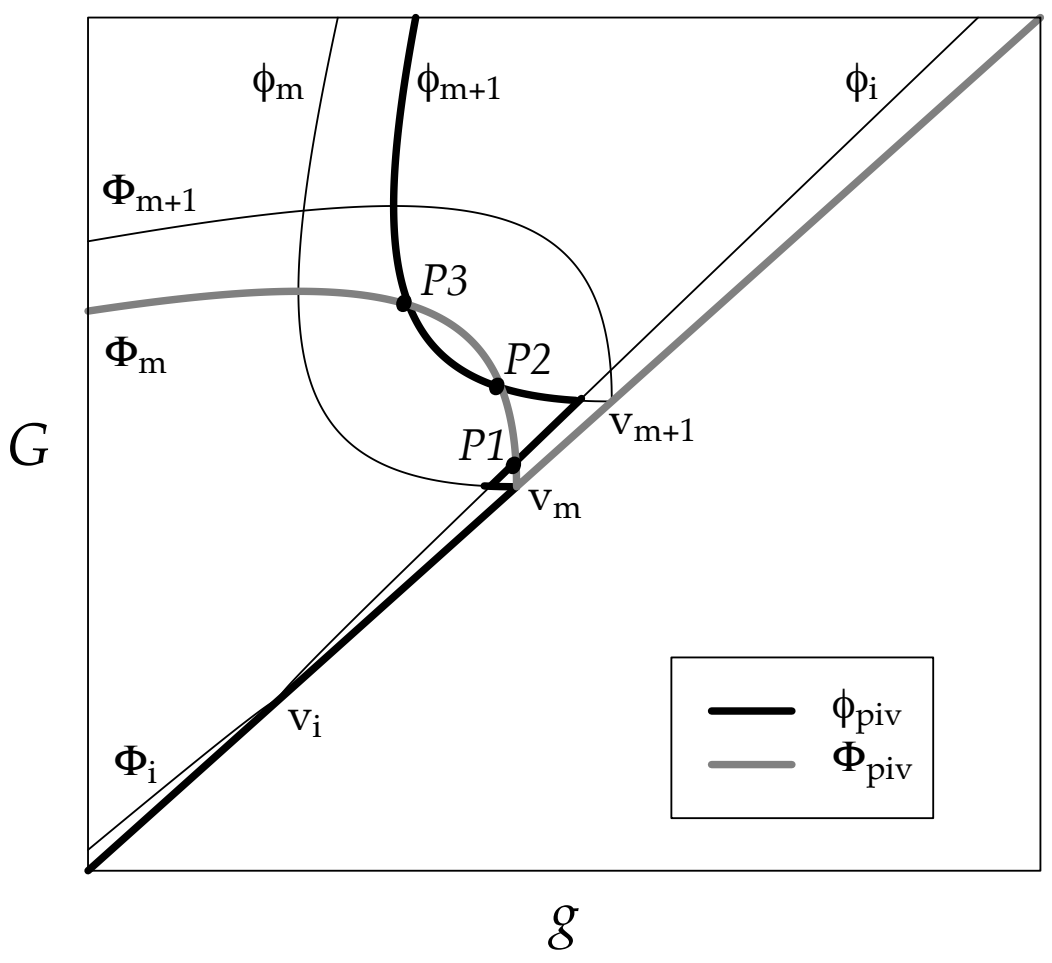

Figure 3. Multiple equilibria exist when $v_{m+1}-v_{m}$ is small. The hasty equilibrium is $P 1$ in this figure; there are two other more patient equilibria, $P 2$ and $P 3$. The figure is drawn using the same parameters as in Figure 4(c), but with $v_{m+1}-v_{m}=0.4$ instead of 0.6 . When $v_{m+1}-v_{m}$ becomes larger, the best-response functions $\phi_{m+1}$ and $\Phi_{m}$ are pulled apart, and eventually do not intersect. Then, the hasty equilibrium P1 remains the only equilibrium.

if they expect others are taking their time, but they may also rush to a decision to preempt others from reaching a different decision in a hurry (a phenomenon we call hasty equilibrium). We discuss these types of equilibria in detail and return to the issue of multiplicity in the next section. When there are multiple equilibria, the equilibrium waiting regions are nested; that is, the most patient equilibrium waiting region contains all other equilibrium waiting regions while the least patient equilibrium waiting region is contained in all others.

Moderation in Committees with Homogeneous Time Preferences. It is useful to point out a feature of equilibrium when all agents have the same time preference. In this case, recalling that $v_{1}<\ldots<v_{2 m-1}$, it can be shown that the equilibrium waiting region $(\hat{g}, \hat{G})$ under decision rule $k$ is the same as the equilibrium waiting region under unanim- 
ity rule for a two-agent group consisting of agents $2 m-k$ and $k$. Furthermore, $\hat{g} \geq g_{2 m-k}^{*}$ and $\hat{G} \leq G_{k}^{*}$, with equality if and only if $k=m$. Because one-sided best-response functions do not cross when agents have the same discount rate, agent $2 m-k$ is pivotal for adopting $\beta$, while agent $k$ is pivotal for $\alpha$. The exact intensity of the static preferences of the remaining agents do not matter for the determination of the equilibrium. This also means that, in the case of identical time preferences, under majority rule, the equilibrium waiting region coincides with the optimal waiting region for the median agent. Under super-majority rule, the pivotal agent for $\alpha$ (i.e., agent $k$ ) adopts $\alpha$ before reaching the point that she deems optimal. Recall that agent $k$ has a stronger preference for $\beta$ than agent $2 m-k$ does. If agent $k$ were to make the decision alone, she would adopt $\alpha$ only when the belief is sufficiently strong to reach $G_{k}^{*}$. The fact that $\hat{G}<G_{k}^{*}$ means that group decision-making leads to a moderation effect: the two pivotal agents choose thresholds that are less extreme than those they would choose were they in full control of the deliberation process. This follows from strategic substitution. Because agent $2 m-k$ prefers to adopt $\beta$ later than agent $k$ does, agent $k$ 's continuation value from waiting is lowered, and she accommodates agent $2 m-k$ by adopting $\alpha$ earlier. ${ }^{11}$

\section{The Drawbacks of Majority Rule}

In our deliberation process, agents are making two related decisions: which alternative to adopt, and how long to wait. The static preference $v_{i}$ is related to the first decision, and the time preference $r_{i}$ is related to the second. When there is two-dimensional heterogeneity, the median voter theorem does not apply; that is, how long the group waits does not depend only on the median discount rate. Because quick decisions are strategic complements, the presence of an impatient agent may cause other agents to stop deliberations early. This, together with the fact that under majority rule it takes only one vote to change the outcome from one alternative to another, implies that the most impatient agent tends to have a disproportionate influence on the length of collective deliberations. This is a potential drawback of majority rule and a useful illustration of the consequences of two-dimensional heterogeneity.

Figure 4 illustrates how the presence of an impatient agent affects equilibrium outcomes. Agent 1 becomes increasingly impatient across the three panels. Despite having a weaker preference for $\beta$ than other agents, agent 1 is willing to adopt decision $\beta$ before the other agents do as $r_{1}$ increases. He becomes pivotal for $\beta$ when $r_{1}$ increases from panel (a) to panel (b) of Figure 4. Agent 2 responds by stopping earlier to adopt $\alpha$, because she expects the impatient agent would stop early to adopt $\beta$. The waiting region of equilibrium $P 2$ is narrower than that of equilibrium $P 1$. One may think of the impatient

\footnotetext{
${ }^{11}$ When agents have different time preferences, we can no longer identify the pivotal agents based on their static preferences alone. Nevertheless, a generalized form of this moderation effect still holds. In any equilibrium under decision rule $k$, the lower equilibrium threshold is higher than the $k$-th highest $g_{i}^{*}$ and the upper equilibrium threshold is lower than the $k$-th lowest $G_{i}^{*}$.
} 
agent 1 as a "swing voter." While he prefers $\alpha$, he is willing to settle for $\beta$ as long as the evidence swings a bit in favor of $\beta$. His vote, therefore, easily switches between $\alpha$ and $\beta$. Hence, both agent 2 , who prefers $\alpha$, and agent 3 , who prefers $\beta$, have an incentive to stop deliberations early to prevent agent 1 from switching sides. These effects are magnified in equilibrium through strategic complementarity.

In panel (b) of Figure 4 , agent 1 is both more impatient and has a stronger preference for $\alpha$ than the other agents. Nevertheless, he continues to influence the deliberation outcome as $r_{1}$ increases further. In panel (c), $\phi_{1}$ approaches the 45-degree line, and the equilibrium $P 3$ is near $\left(v_{m}, v_{m}\right)$. In this equilibrium, although the impatient agent 1 has a stronger preference for $\alpha$ than other agents, he votes for $\beta$ as soon as the belief drops slightly below the threshold for $\alpha$ to avoid further delay. In contrast, although agent 2 is patient, she votes for $\alpha$ as soon as the belief goes slightly above her static preference. The value of waiting is low for her because she is almost indifferent between $\alpha$ and $\beta$ at the belief under which $\beta$ is adopted.

In Figure 4 there are only three agents, but the same logic applies regardless of the size of the group and their preferences.

Proposition 2. Under majority rule, there is an equilibrium in which deliberation ends arbitrarily quickly when one agent is sufficiently impatient: for any $\varepsilon>0$ there exists $\bar{r}(\varepsilon)$ such that if $r_{i} \geq \bar{r}(\varepsilon)$ for some agent $i$, then there is an equilibrium waiting region $(\hat{g}, \hat{G})$ with width less than $\varepsilon$ and with $v_{m} \in(\hat{g}, \hat{G})$.

We call the type of equilibrium highlighted in Proposition 2, one in which the waiting region is no wider than $\varepsilon$, an $\varepsilon$-hasty equilibrium. For vanishingly small $\varepsilon$, in an $\varepsilon$-hasty equilibrium, the group decides almost immediately, and which alternative is chosen depends only on whether the initial belief $\theta_{0}$ is greater or lower than the static preference of agent $m$. The equilibrium outcome is, therefore, almost identical to that of a static problem in which the median voter decides without the benefit of any information collection. Note that the effect of the impatient agent on the equilibrium outcome cannot be counterbalanced by the presence of one or more extremely patient agents in the group. In Proposition 2, $\bar{r}(\varepsilon)$-the discount rate that ensures an equilibrium with an $\varepsilon$-waiting region-does not depend on the preferences of the other agents. Hence, the welfare loss to the other agents could be large. Under majority rule, one agent is enough to trigger a hasty equilibrium outcome, even when every other agent is very patient. In this sense, majority rule is not a robust decision-making mechanism because the length of deliberation can be dominated by one agent with extreme time preference. ${ }^{12}$

As long as there is a very impatient agent, a hasty equilibrium exists. Whether the

\footnotetext{
${ }^{12}$ Since the agents' utility functions are continuous in cutoffs, an equilibrium of the continuous-time model would be an epsilon-Nash equilibrium of the discrete-time model with short time periods. When there is a very impatient agent, we can verify that there is a mixed-strategy discrete-time equilibrium outcome close to the hasty equilibrium outcome in the continuous time model.
} 


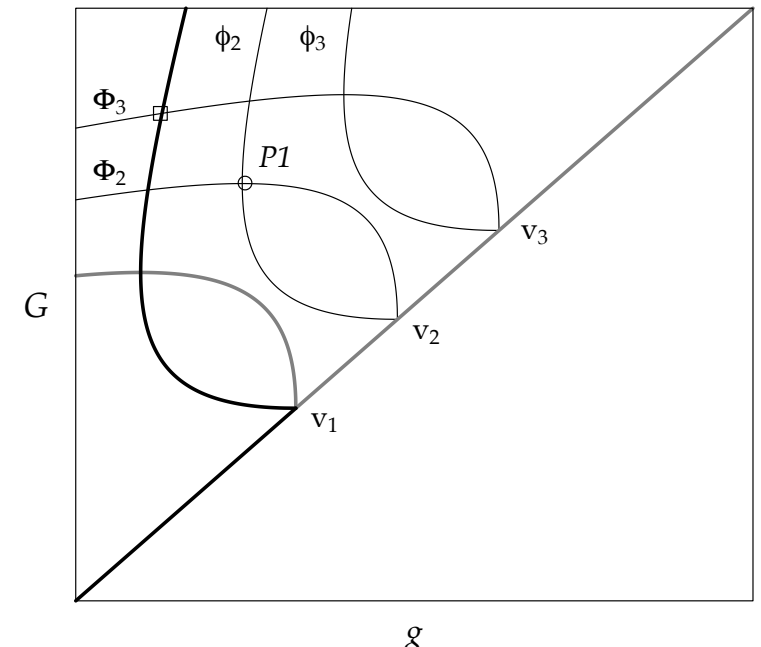

(a) $r_{1}=r_{2}=r_{3}$

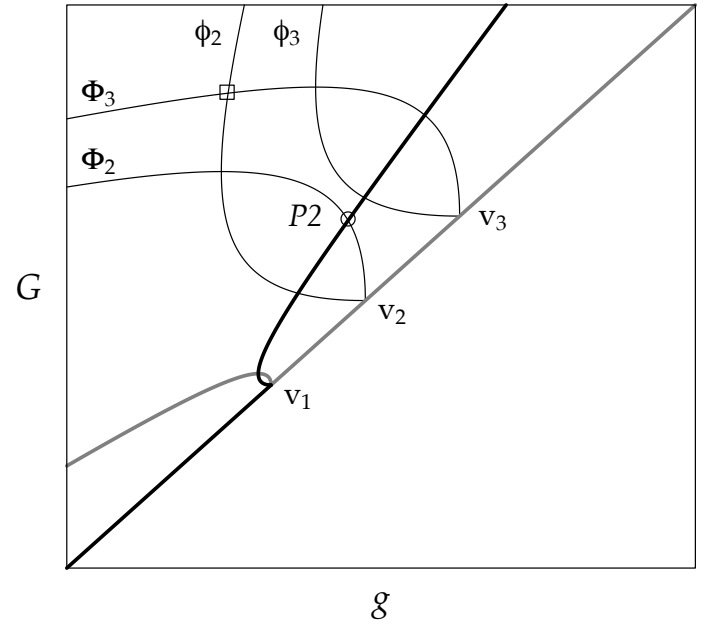

(b) $r_{1}>r_{2}=r_{3}$

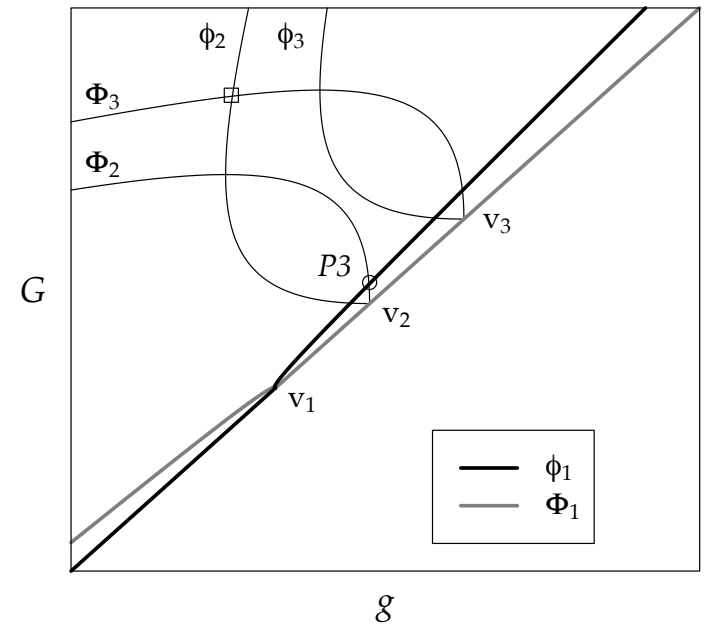

(c) $r_{1}>>r_{2}=r_{3}$

Figure 4. Deliberation collapses as one of the agents becomes extremely impatient. When $r_{1}=r_{2}=r_{3}=$ 0.039 (panel (a)), agent 1 is not pivotal and the equilibrium is $P 1$. If $r_{1}$ increases to 0.6 , his best-response function $\phi_{1}$ bends toward the 45-degree line. Agent 1 becomes pivotal and the equilibrium is $P 2$. If $r_{1}$ increases further to 9 (panel (c)), $\phi_{1}$ bends further and the equilibrium P3 is very close to the 45-degree line. The implied waiting region is very short. The circles in each panel indicate the respective equilibrium points when the decision rule requires unanimity. 
equilibrium is unique depends on whether the preferences of the other agents are sufficiently different. In Figures 3 and 4 , all agents except the impatient agent $i<m$ have the same discount rate. In Figure 3, the difference between $v_{m+1}$ and $v_{m}$ is small, and there exist two other equilibria that are more patient than the hasty equilibrium. In Figure $4(\mathrm{c})$, the difference between $v_{m+1}$ and $v_{m}$ is large, and the hasty equilibrium is the unique equilibrium.

When multiple equilibria exist, these equilibrium outcomes cannot be Pareto ranked. Different agents may have different preferences over different equilibria. More importantly, even the same agent may have different preferences over two equilibria at different beliefs. For example, an agent with strong static preference for $\alpha$ would prefer a hasty equilibrium when the belief is high (so she can obtain $\alpha$ immediately), but would prefer a more patient equilibrium when the belief is low (so she will not get $\beta$ immediately). Thus, even if a majority of agents initially prefer a more patient equilibrium to a hasty equilibrium, some of these agents may change their minds as the belief changes. ${ }^{13}$

Compared to majority rule, super-majority rules are more resistant to the excessive influence of a few impatient agents. In Figure 4(c), if unanimity rule is used instead of majority rule, the equilibrium, now given by the intersection of $\phi_{2}$ and $\Phi_{3}$, will no longer be a hasty equilibrium. There is no need under unanimity rule for agents 2 and 3 to rush to a decision because the vote of the impatient voter is no longer sufficient to swing the outcome. We now elaborate on the robustness of super-majority against the presence of impatient agents.

Define the number of requisite swing votes of a rule as the minimum number of vote changes to shift the outcome from adopting one alternative immediately to adopting the other alternative immediately. The number of requisite swing votes of majority rule is 1 -if $\alpha$ and $\beta$ each receive $m$ votes, the last vote determines the outcome. More generally, under rule $k$ an alternative must receive at least $k$ votes to be adopted. Because there are $2 m-1$ agents altogether, the number of requisite swing votes of rule $k$ is $2 k-(2 m-1)$.

In what follows, we say that a group of impatient agents can trigger a collapse of deliberation if, for any $\varepsilon>0$, there is an equilibrium with a waiting region no wider than $\varepsilon$ when every agent in that group has a discount rate greater than some $\tilde{r}(\varepsilon)$, regardless of the preferences of other agents.

Proposition 3. A group of impatient agents cannot trigger a collapse of deliberation whenever the size of the group is smaller than the number of requisite swing votes.

\footnotetext{
${ }^{13}$ The result that one very impatient agent can trigger hasty deliberations depends on two features of our model: first, information arrives continuously; and second, as information arrives an agent's relative preference between the two alternatives may change. In contrast, in the collective search model of Albrecht, Anderson and Vroman (2010), a new proposal is drawn every period, and in equilibrium the agents never adopt a past proposal. In their model an extremely impatient agent is essentially redundant because she will accept almost any proposal.
} 
Under rule $k$, at least $2 k-(2 m-1)$ agents switch their votes from $\alpha$ at the upper cutoff to $\beta$ at the lower cutoff. Each of them can unilaterally delay the adoption of either alternative at the equilibrium threshold. It can be shown that, given $r_{i}$, agent $i$ always prefers delaying the adoption of at least one of the alternatives when the waiting region becomes too narrow. Hence, unless all agents who vote for both alternatives at the cutoffs are very impatient, no hasty equilibrium can exist. By contrast, in a hasty equilibrium under majority rule only the very impatient agent is voting for both $\alpha$ and $\beta$ at the adoption thresholds. Of the remaining $2 m-2$ agents, half vote only for $\alpha$ but not $\beta$, and the other half vote only for $\beta$ but not $\alpha$. While each of these $2 m-2$ agents may like to simultaneously delay the adoption of both alternatives, none of them can do so. ${ }^{14}$

Up to now we have focused on when extreme outcomes (collapse of deliberation) may arise. In evaluating voting rules it is certainly important to understand more generally how the accuracy of decisions (or, conversely their timeliness) responds to changes in the voting rules. ${ }^{15}$ It is also interesting to study the role of the composition of the committee. Some of our analysis is sharper when we assume symmetry within the committee. Formally, a committee is symmetric if $r_{1}=\ldots=r_{2 m-1}$ and $v_{m}-v_{j}=v_{2 m-j}-v_{m}$ for $j=1, \ldots, m-1$. In a symmetric committee, we say that preferences become more diverse when, for any $j=1, \ldots, m-1, v_{j}$ decreases and $v_{2 m-j}$ increases by the same amount. In other words, a more diverse symmetric committee is one in which agents on both sides of the median agent hold stronger opposing preferences for their favored alternative.

\section{Proposition 4.}

1. At least one of the alternatives is adopted later and with a greater accuracy in the most patient and the least patient equilibria when $k$ increases or when $r_{i}$ for any agent $i$ decreases.

2. In any equilibrium under rule $k$, the waiting region is no wider than the equilibrium waiting region under unanimity rule for any subset of $k$ agents.

3. In a symmetric committee both alternatives are adopted later and with a greater accuracy when all agents become more patient, when the size of the majority requirement increases, or when the static preferences of agents become more diverse.

It is easiest to glean intuition into part 3 of the proposition. Since the pivotal agents in a symmetric committee are agents $2 m-k$ and $k$ (see the discussion at the end of Section

\footnotetext{
${ }^{14}$ Although we consider decision rules that treat the two alternatives symmetrically, we note that the issues we describe also exist in the case of asymmetric decision rules. For a rule that requires $k_{\alpha}$ votes to adopt $\alpha$ and $k_{\beta}$ votes to adopt $\beta$, the number of requisite swing votes is $k_{\alpha}+k_{\beta}-(2 m-1)$. As in the symmetric case, one impatient agent may trigger a collapse of deliberation if the number of requisite swing votes is one and, conversely, a group of impatient agents cannot trigger a collapse of deliberation if its size is smaller than the number of requisite swing votes. We thank a referee for raising this point.

${ }^{15}$ The accuracy of a decision reflects the probability with which the alternative that all agents prefer in the realized state is selected. We say that an aternative is adopted with greater accuracy in one setting relative to another if the following holds: When that alternative is selected in the first setting, the probability it is optimal given the realized state is higher than when it is selected in the second setting.
} 
4), raising the majority requirement from $k$ to $k^{\prime}$ has the same effect as making preferences in the outcome-equivalent two-person jury more diverse (raising the static preference of one juror from $v_{2 m-k}$ to $v_{2 m-k^{\prime}}$, while raising that of another from $v_{k}$ to $\left.v_{k^{\prime}}\right)$. In part 3 of Proposition 4, we show that such a change will expand the equilibrium waiting region (i.e., lower $\hat{g}$ and raise $\hat{G}$ ), as each of the pivotal agents demands greater evidence before adopting the alternative she is biased against. Deliberations are longer because, starting from any initial belief within the waiting region, the time it takes to reach the boundaries increases. Decisions are more accurate because $\alpha$ is adopted only when the belief reaches a higher upper cutoff $\hat{G}$, which corresponds to a higher probability that the state is $A$. Likewise, a lower equilibrium $\hat{g}$ means that $\beta$ is adopted at a point when the probability of state $B$ is higher. This result provides a contrast between our characterization and those pertaining to private information collection, as in Feddersen and Pesendorfer (1998), Persico (2004), and Austen-Smith and Feddersen $(2005,2006)$.

A symmetric committee provides a particularly simple setting to study the effect of raising the size of the majority requirement $k$. Symmetry is sufficient but not necessary for part 3 of Proposition 4 to hold. However, large departures from symmetry may invalidate the result that an increase in $k$ always produces more accurate decisions. Imagine, for example, that agent $2 m-k^{\prime}$ is much more biased for $\alpha$ than agent $2 m-k$, while agent $k^{\prime}$ is only slightly more biased for $\beta$ than agent $k$. When the decision rule is raised from $k$ to $k^{\prime}$, each of the two pivotal agents under decision rule $k^{\prime}$ wants to extend the threshold before adopting the alternative they control. Because slow decisions are strategic substitutes, the less extreme agent $k^{\prime}$ may want to accommodate the longer expected waiting time caused by the other more extreme pivotal agent by adopting $\alpha$ earlier. If the second effect dominates, then it is possible for the equilibrium upper cutoff $\hat{G}$ to decrease, making the decision for $\alpha$ less accurate when it is adopted. In the general case where preferences are not symmetric or when there is two-dimensional heterogeneity, it is not always the case that increasing $k$ lowers $\hat{g}$ and raises $\hat{G}$. Nevertheless, part 1 shows that the waiting region always become wider when $k$ increases or when $r_{i}$ decreases for some agent $i$. This implies that at least one of the alternatives will be adopted later and with a greater accuracy.

Part 2 of Proposition 4 provides an interesting contrast with Proposition 3: the effects of very impatient agents and very patient agents are not symmetric. Voting to delay an alternative by a very patient agent would not cause other agents to adopt the other alternative later. Unless they can block a decision by themselves, a subset of very patient agents cannot prevent the group from reaching a decision. It takes $2 m-k$ votes to block a decision under rule $k$. As the majority requirement is increased by one, the maximum number of very patient agents that can be allowed without causing endless deliberations falls by one. In contrast, the number of requisite swing votes increases by two. Hence, a properly chosen super-majority rule is robust to the presence of a few extreme agents whose discount rates are either very high or very low. For example, suppose the very 
impatient agents and the very patient agents each make up $1 / 4$ of the size of the group. Then a decision rule with less than $3 / 4$ super-majority requirement would prevent the patient agents from creating protracted deliberations, and the impatient agents cannot trigger a collapse of deliberation as long as the super-majority requirement is greater than $5 / 8$.

\section{Advantages of Majority Rule}

We now turn to the advantages of majority rule. We highlight two important benefits: in many environments majority rule is preferred by the committee members themselves and majority rule respects static preferences.

We now consider how committee members evaluate voting rules and their associated the accuracy-timeliness trade-off. We can obtain a particularly clean result maintaining the assumption of symmetry. Because all agents have the same time preference, the median agent is pivotal for both $\alpha$ and $\beta$ under majority rule. The equilibrium waiting region is simply her optimal waiting region $\left(g_{m}^{*}, G_{m}^{*}\right)$. Let $(\hat{g}, \hat{G})$ represent the equilibrium waiting region under super-majority rule $k$. Proposition 4 shows that $\hat{G}-\hat{g}>G_{m}^{*}-g_{m}^{*}$. Furthermore, by symmetry, these two equilibrium waiting regions are both centered at $v_{m}$. We call a committee unbiased if its preference profile satisfies symmetry, and if the initial belief $\theta_{0}$ equals $v_{m}$.

Proposition 5. In an unbiased committee, all agents prefer majority rule to super-majority rule.

This result says that, if the initial beliefs are not biased in favor of a particular alternative, then there is no conflict in the committee as to how to resolve the accuracy-timeliness tradeoff. From the perspective of agent $m$, it is obvious that the waiting region $\left(g_{m}^{*}, G_{m}^{*}\right)$ is preferable to $(\hat{g}, \hat{G})$. From the perspective of any other agent $j \neq m$, the ideal waiting region is centered at $v_{j}$. Thus, both majority rule and super-majority rule generate a waiting region that is centered at the "wrong" place for agent $j$. But since agent $j$ has the same time discount rate as agent $m$, super-majority rule also produces a waiting region that is "too wide." This does not necessarily mean that agent $j$ prefers majority rule to supermajority rule, because agent $j$ 's utility also depends on the initial belief $\theta_{0}$. However, when the initial belief is near the center of the waiting region, there is unanimity in favor of majority rule: all agents deem the waiting region under super-majority rule too wide and prefer to use majority rule. Equivalently, in an unbiased committee, all agents prefer to delegate the decision-making power to the median voter. ${ }^{16}$ In the proof, we show that, just as in the case of part 3 of Proposition 4, the result is robust to small violations of symmetry.

If, on the other hand, the initial belief $\theta_{0}$ is greater than $G_{m}^{*}$ but smaller than $\hat{G}$, majority

\footnotetext{
${ }^{16}$ Indeed, majority rule would generate the same outcomes as the median voter would by behaving optimally as a single decision-maker.
} 
rule would have adopted $\alpha$ immediately, while there is still some prospect of getting $\beta$ under super-majority rule. Thus, an agent $j$ with a sufficiently strong preference for $\beta$ may prefer super-majority rule to majority rule despite the longer wait. However, in this example all agents with a preference for $\alpha$ stronger than that of the median agent prefer to adopt $\alpha$ immediately than to wait at this belief $\theta_{0}$. In fact, we can show that, in general, even though the preference for majority rule may not be unanimous, there is always a majority of group members who prefer majority rule to a super-majority rule.

Propositions 4 and 5 suggest that, while symmetric committees themselves prefer majority rules, the quality of decisions is actually superior with more restrictive supermajority rules. That increased quality comes at the cost of longer deliberation, which is borne by the agents themselves. Whenever there are positive externalities from high quality collective decisions, which may be especially relevant for juries or standard-setting committees since their decisions arguably impact a society of individuals who do not bear the deliberation cost themselves, super-majority rules may be preferable.

In the more general case of two-dimensional heterogeneity and asymmetric committees, there is an additional concern that arises when using super-majority rules. One criterion for evaluating the quality of a collective decision is whether it accords with the static preferences of members of the group. Under majority rule, if a majority of agents prefer a different alternative to the one adopted in equilibrium, they can simply adopt the one they prefer. This simple reasoning immediately implies that majority rule respects static preferences, in the sense that at the end of deliberations at least a majority of agents favor the alternative chosen to the one not chosen.

Proposition 6. Majority rule produces outcomes that always respect static preferences, i.e., $v_{m} \in$ $(\hat{g}, \hat{G})$. In contrast, under super-majority rule $k>m$, there is always a preference profile where as many as $k-1$ agents prefer $\alpha$ to $\beta$ at a region in which $\beta$ is selected: for a fixed profile of discount rates and static preferences of agents $1, \ldots, k-1$, there exists $\underline{v}$ such that, if $v_{i}>\underline{v}$ for $i \geq k$, then $\hat{g}>v_{k-1}$.

Figure 5 illustrates. Panel (a) shows a symmetric committee with three agents under unanimity rule. The equilibrium waiting region $(\hat{g}, \hat{G})$ contains $v_{m}$. That is, when $\beta$ is adopted at $\hat{g}$, agent $m$ and agent 3 (a majority) both prefer the adopted alternative to the one not chosen. However, when agent 3 has extreme preference for $\beta$ ( $v_{3}$ is large compared to static preference parameters of other agents), the equilibrium $\hat{g}$ is greater than $v_{m}$, meaning that both agent 1 and agent $m$ (a majority) actually prefer $\alpha$ to $\beta$ despite voting for $\beta$ at that cutoff.

Intuitively, under super-majority rules, a majority of agents may vote for $\beta$ when they prefer $\alpha$ in order to get a faster decision. Thus, having a greater majority requirement does not always help build consensus. Although a greater majority requirement may create the appearance of a greater consensus, some of those who vote for an alternative 


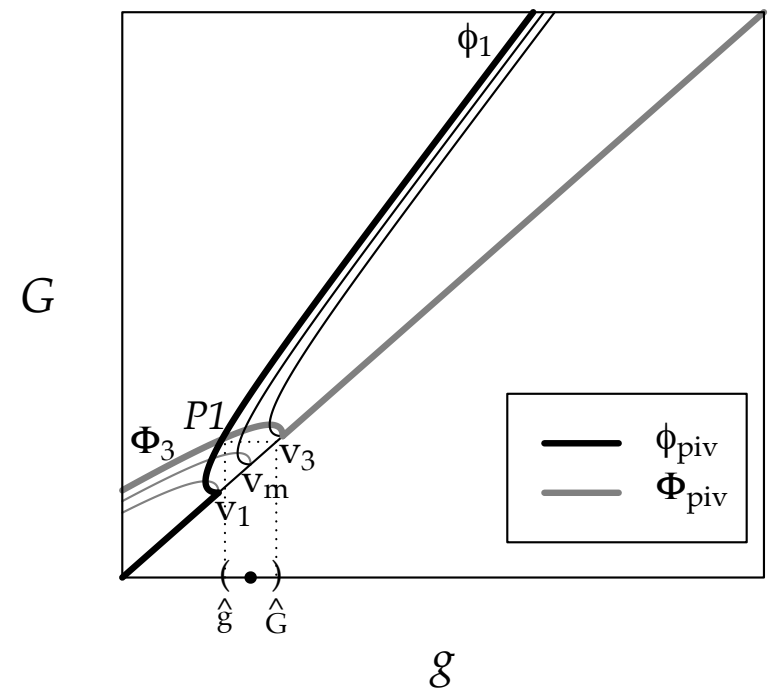

(a) $v_{m} \in(\hat{g}, \hat{G})$

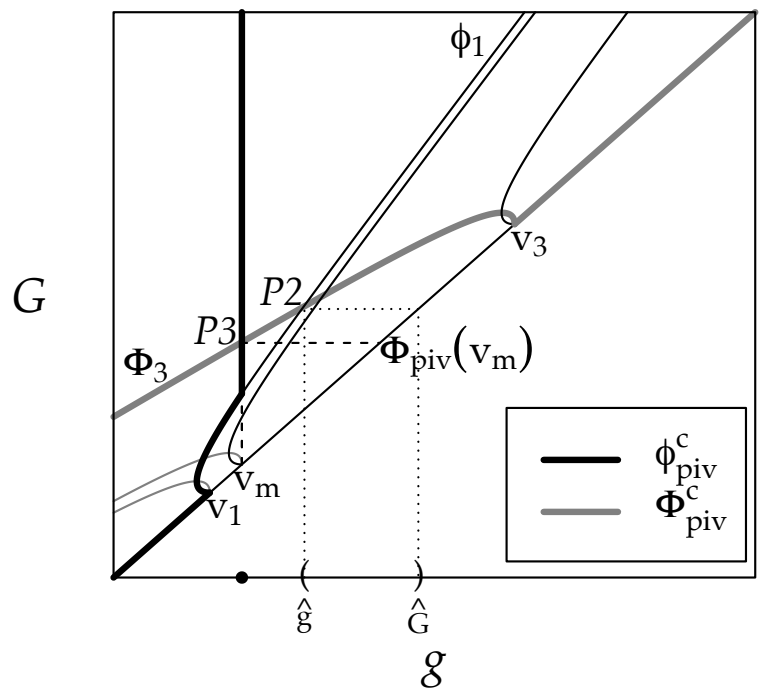

(b) $v_{m} \notin(\hat{g}, \hat{G})$

Figure 5. Three agents make a decision under unanimity rule. In panel (a), static preferences are not far apart, and equilibrium $P 1$ produces outcomes that respect static preferences. In panel $(b)$, agent 3 is extremely biased for $\beta$, and both agent 1 and agent $m$ actually prefer $\alpha$ to $\beta$ when $\beta$ is adopted at $\hat{g}$ in equilibrium P2. A two-stage decision process with rule $k_{D}=3, k_{d}=2$ moves the equilibrium outcome from $P 2$ to $P 3$. The equilibrium waiting region is $\left(v_{m}, \Phi_{\text {piv }}\left(v_{m}\right)\right)$. Deliberation stops at $v_{m}$ and, at the decision stage, agents $m$ and 3 vote to adopt $\beta$. However, the waiting region is also wider (P3 is farther from the 45-degree line than is P2). In panel $(a),\left(v_{1}, v_{m}, v_{3}\right)=(-0.2,0,0.2)$ and $r_{1}=r_{m}=r_{3}=0.039$. In panel $(b), v_{3}$ is changed to 1.7 .

may merely be trying to avoid costly delay. ${ }^{17}$

\section{Two-Stage Process: Deliberation Rules and Decision Rules}

In our analysis thus far, a single rule $k$ affects two types of decisions: how long to acquire information, and what action to take once information collection comes to an end. It is natural to consider the consequences of a two-stage procedure that distinguishes between a deliberation rule and a decision rule. A special case of a deliberation rule in a jury setting corresponds to repeated straw polls, with a final vote taken according to the decision rule once the outcome of the straw poll indicates that sufficient consensus has been achieved. ${ }^{18}$

\footnotetext{
${ }^{17}$ In The Federalist No. 58, James Madison explained why he did not favor super-majority rules: "It would be no longer the majority that would rule; the power would be transferred to the minority. ... [A]n interested minority might take advantage of it to screen themselves from equitable sacrifices to the general weal, or in particular emergencies to extort unreasonable indulgences" (Hamilton, Madison, and Jay 1982, pp. 298-299).

${ }^{18}$ The guidelines distributed to jurors in many U.S. courts indicate that this protocol reflects the deliberation process suggested to juries, see Murphy and Boatright (1999a, 1999b).
} 
In general, deliberation rules may differ from decision rules. For instance, many committees feature a committee chairman who has the same power as all the other members over action decisions, but has a special role to play (and more power) in deliberation decisions. The chairman case can be modeled as either a dictator at the deliberation stage, or as the median voter in the committee.

We model the deliberation rule as a threshold rule $k_{D}$ such that deliberation ends as soon as $k_{D}$ members of the committee vote to end deliberation. The decision rule is analogously captured by a rule $k_{d}$ that describes the specific qualified majority required for reaching a decision about which action to take after deliberation stops. In a two-stage process, it might be the case that at the decision stage, there is no $k_{d}$-majority of votes for either alternative. In this case, we say that the committee is indecisive. We assume that when the committee is indecisive, $\alpha$ or $\beta$ is determined by the flip of a fair coin. ${ }^{19}$ A classic example of an indecisive committee corresponds to a hung jury that does not reach the quorum required for conviction or for acquittal.

The analysis of two-stage processes allows us to highlight two distinct points. The first point pertains to whether outcomes respect static preferences. We saw that, under super-majority rules, in the one-stage process it is possible that agents may vote against their static preferences in order to shorten deliberation. This is no longer possible in a two-stage process because the vote on the decision is separated from the vote on ending deliberation; once a successful vote to end deliberation has taken place, no agent has any incentive to vote against her static preference. This feature of the two-stage process thus prevents a minority of agents from gaining excessive leverage under super-majority rules. In turn, in this setting super-majority rules in the deliberation stage may become more attractive from a societal perspective, as they still retain their function of promoting longer deliberation. The second point pertains to the relative importance of deliberation rules and decision rules for the time it takes committees to make a decision and for the accuracy of these decisions.

In order to tackle the first point, we assume that the decision rule $k_{d}$ is majority rule, which simplifies the analysis considerably since the committee is never indecisive in the second stage. However, we make no restrictions on the deliberation rule $k_{D}$. In the second stage, we assume that each agent votes for the alternative she likes given the current belief $\theta$. Hence, $\alpha$ is adopted if $\theta \geq v_{m}$ and $\beta$ is adopted when $\theta<v_{m}$. This is often referred to as "sincere voting" and results from ruling out weakly dominated strategies at the subgame corresponding to the decision stage. In the first stage, each agent knows that voting for ending deliberation when $\theta \geq v_{m}$ is tantamount to voting for $\alpha$. Therefore, instead of analyzing the two-stage game per-se, we study a modification of the one-stage game, which we call the constrained deliberation game. In the constrained deliberation game, as

\footnotetext{
${ }^{19}$ The exact assumption we make about the consequences of indecisive committees is inconsequential as most of our analysis focuses on cases where these do not occur.
} 
before, at every instant $t$, each agent independently votes for $\alpha, \beta$, or neither, and an alternative is adopted at time $t$ if it receives $k_{D}$ votes or more. The constraint is that agents can vote for $\alpha$ only when $\theta \geq v_{m}$ and for $\beta$ only when $\theta<v_{m}$. Because of these constraints, we need to slightly modify the second part of our definition of equilibrium in section 2. Instead of requiring each agent to vote for an alternative before the belief reaches the equilibrium cutoff whenever the marginal gain from an earlier decision is positive, we now only require an agent to do so when the constraints are not binding. In what follows, we slightly abuse language by referring to equilibria of this constrained deliberation game as the outcomes of the two-stage process described above.

Recall that for any agent $i$, we denoted by $\phi_{i}(G)$ the agent's lower best-response function, the optimal lower cutoff $g$ for any fixed upper cutoff $G$. In the constrained game, the agent cannot vote for $\beta$ when $\theta \geq v_{m}$. Therefore, her constrained best response would be given by $\min \left\{\phi_{i}(G), v_{m}\right\}$. A similar calculation follows for the agent's constrained upper best response. We denote the constrained best-response functions for agent $i$ as follows:

$$
\begin{aligned}
\phi_{i}^{c}(G) & \equiv \min \left\{\phi_{i}(G), v_{m}\right\}, \\
\Phi_{i}^{c}(g) & \equiv \max \left\{\Phi_{i}(g), v_{m}\right\} .
\end{aligned}
$$

Define $\phi_{p i v}^{c}\left(G ; k_{D}\right)$ to be the $k_{D}$-th largest $\phi_{i}^{c}(G)$ for each $G$, and $\Phi_{p i v}^{c}\left(g ; k_{D}\right)$ to be the $k_{D}$-th smallest $\Phi_{i}^{c}(g)$ for each $g$. Using the same reasoning as in the unconstrained case, it is straightforward to see that $\left(\hat{g}^{c}, \hat{G}^{c}\right)$ is an equilibrium outcome of the constrained deliberation game, if and only if it is a fixed point of $\left(\phi_{p i v}^{c}, \Phi_{p i v}^{c}\right)$ and $\hat{g}^{c}<\hat{G}^{c}$.

There are two types of equilibrium outcomes in this constrained deliberation game, and therefore in the two-stage process. An unconstrained outcome is one in which neither cutoff is constrained (i.e., $\hat{g}^{c}<v_{m}<\hat{G}^{c}$ ). A constrained outcome is one in which one of the equilibrium cutoffs is $v_{m}$. It is straightforward to show that for any $g$ and $G$

$$
\begin{aligned}
\phi_{p i v}^{c}\left(G ; k_{D}\right) & =\min \left\{\phi_{p i v}(G), v_{m}\right\}, \\
\Phi_{p i v}^{c}\left(g ; k_{D}\right) & =\max \left\{\Phi_{p i v}(g), v_{m}\right\} .
\end{aligned}
$$

Hence, an unconstrained outcome is an equilibrium outcome under the two-stage decision process if and only if it is also an equilibrium outcome in the unconstrained deliberation game.

When an equilibrium outcome in the unconstrained deliberation game does not respect static preferences, it can no longer be implemented under the two-stage decision process.

A constrained outcome $\left(\hat{g}^{c}, \hat{G}^{c}\right)$ is an equilibrium of the two-stage process if and only if $\hat{g}^{c}<\hat{G}^{c}$ and one of the following conditions holds: 
1. $\left(\hat{g}^{c}, \hat{G}^{c}\right)=\left(v_{m}, \Phi_{p i v}\left(v_{m}\right)\right)$, and $\phi_{p i v}\left(\Phi_{p i v}\left(v_{m}\right)\right) \geq v_{m} ;$

2. $\left(\hat{g}^{c}, \hat{G}^{c}\right)=\left(\phi_{p i v}\left(v_{m}\right), v_{m}\right)$, and $\Phi_{p i v}\left(\phi_{p i v}\left(v_{m}\right)\right) \leq v_{m}$.

Indeed, a constrained outcome is constrained either on the lower or the upper cutoff. Condition 1 corresponds to the former, where the lower cutoff is constrained: $\hat{g}^{c}=v_{m}$. To see the intuition for this condition, notice that the upper cutoff must be a best response for the corresponding pivotal agent, and so it must be the case that $\hat{G}^{c}=\Phi_{p i v}\left(v_{m}\right)$. Furthermore, the lower cutoff must be a constrained best response for the relevant pivotal agent, so that

$$
\phi_{p i v}^{c}\left(\hat{G}^{c} ; k_{D}\right)=\min \left\{\phi_{p i v}\left(\hat{G}^{c}\right), v_{m}\right\}=\min \left\{\phi_{p i v}\left(\Phi_{p i v}\left(v_{m}\right)\right), v_{m}\right\}=v_{m} .
$$

It follows that $\phi_{p i v}\left(\Phi_{p i v}\left(v_{m}\right)\right) \geq v_{m}$. Analogous considerations pertain to outcomes with a constrained upper cutoff, generating condition 2 above.

Panel (b) of Figure 5 depicts a constrained equilibrium with three agents. In the figure, the equilibrium outcome in the unconstrained game under unanimity rule is $(\hat{g}, \hat{G})$ (point $P 2)$. In this equilibrium agents 1 and $m$ prefer $\alpha$ but vote to adopt $\beta$ at $\hat{g}$. Under the two-stage decision process the equilibrium outcome is $\left(v_{m}, \Phi_{\text {piv }}\left(v_{m}\right)\right)$ (point $P 3$ ). Notice that all three agents prefer to adopt $\beta$ earlier but cannot vote for $\beta$ until $\theta$ reaches $v_{m}$. Intuitively, $P 2$ is not an equilibrium outcome in a two-stage decision process because if deliberation ends at $\hat{g}$, agents 1 and $m$ will vote for $\alpha$ in the second stage. Anticipating that, agent 3 , who prefers waiting at $\hat{g}$, would hold on until either agent $m$ switches and supports $\beta$ (when $\theta=v_{m}$ ) or she switches and prefers $\alpha$ to waiting (when $\theta=\Phi_{p i v}\left(v_{m}\right)$ ). From panel (b) of Figure 5 it is clear that the waiting region of a constrained equilibrium is wider than that of the equilibrium it replaces in the unconstrained game, and hence the corresponding decisions taken by the committee are more accurate. In fact, this is generally the case, and we have the following proposition.

Proposition 7. Consider an equilibrium outcome $(\hat{g}, \hat{G})$ of the one-stage process for a committee with preference profile $\left(v_{1}, \ldots, v_{2 m-1}\right)$ and voting rule $k$. If either $\hat{g} \geq v_{m}$ or $\hat{G} \leq v_{m}$, then there exists an equilibrium outcome $\left(\hat{g}^{c}, \hat{G}^{c}\right)$ for the same committee in the constrained deliberation game with deliberation rule $k_{D}=k$ such that the waiting region $\left(\hat{g}^{c}, \hat{G}^{c}\right)$ contains $(\hat{g}, \hat{G})$.

The proposition implies that a group of impatient agents cannot trigger a collapse of deliberation in a two-stage process under deliberation rule $k_{D}$ and decision rule $m$ in the constrained game if it cannot do so under the same rule $k=k_{D}$ in the one-stage process. If preferences are symmetric, the one-stage and two-stage processes have the same set of equilibrium outcomes. But when preferences are unbalanced, having a separate decision vote offers additional protection against outcomes dominated by extreme minority preferences. This protection, however, is not without cost: since the length of deliberation is likely to be longer, committee members may actually prefer the unconstrained equilibrium to the constrained one. 
We now turn to the relative impacts of deliberation and decision rules. We allow any decision rule and any deliberation rule. The equilibrium notion we use is that of Nash equilibrium with strategies that are not weakly dominated at any subgame corresponding to the decision stage. We focus on scenarios in which all agents are very patient. It is easy to see that in any equilibrium of the one-stage deliberation game, when agents are sufficiently patient, the waiting region must contain the static preference $v_{i}$ for all agents $i$. This means that when an alternative is adopted at the cutoff, it is unanimously preferred by all agents. We show that the resulting unconstrained outcome of the one-stage game is an equilibrium outcome of the two-stage decision process, regardless of the second-stage decision rule $k_{d}{ }^{20}$ In particular, neither the time to a decision nor the relative probability of the two types of errors depend on the decision rule.

Proposition 8. Consider any profile of static preferences and any two-stage decision rule $\left(k_{D}, k_{d}\right)$. There exists $\underline{r}$ such that, whenever $r_{i} \leq \underline{r}$ for all $i$, if $(\hat{g}, \hat{G})$ is an equilibrium outcome of the one-stage process under rule $k$, then it is an equilibrium outcome of the two-stage process for deliberation rule $k_{D}=k$ and any decision rule $k_{d}$. Furthermore, in the corresponding equilibrium, voting is unanimous at the decision stage.

Proposition 8 implies that, when agents are sufficiently patient, the decision rule has no influence on certain equilibrium outcomes. Furthermore, these equilibria can be implemented using a one-stage process. In the following section we discuss some empirical and experimental evidence on juries consistent with this result.

\section{Empirical Relevance}

\subsection{Application to Juries}

In this section we discuss some evidence on juries documented in the literature. Some of this evidence speaks to the basic structure of the model and some is directly related to our results. This evidence suggests that our model is broadly consistent with some patterns in the data.

In the model, longer deliberation corresponds to additional signals received by the committee. Our interpretation is that this is a reasonable shortcut for thinking about how deliberation helps jurors gain an understanding of the evidence presented at trial. Even though no additional explicit information is received by the jurors during deliberation, it has the function of processing information and understanding the relative importance of different, potentially contrasting pieces of evidence. ${ }^{21}$ In particular, the model fits a

\footnotetext{
${ }^{20}$ We say that $(g, G)$ is an equilibrium outcome of the two-stage process with voting rules $\left(k_{D}, k_{d}\right)$ if there is an equilibrium of the two stage-process that induces a conclusion of information collection when beliefs are outside of the interval $(g, G)$, a choice of $\alpha$ for beliefs greater than $G$, and a choice of $\beta$ for beliefs lower than $g$.

${ }^{21}$ During deliberation, the jury must sift through the mass of sometimes conflicting evidence presented
} 
plausible deliberation protocol in which the jury conducts repeated straw polls until a sufficient consensus emerges.

Legal scholars emphasize the fact-finding role of juries (e.g., Vidmar and Hans 2007). A substantial fraction of deliberation appears to be devoted to a discussion of the facts (Pennington 1983). In fact, starting from Kalven and Zeisel (1966), numerous scholars have argued that juries do a good job at reaching an understanding of the facts. In a study of mock juries, Ellsworth (1989) writes: "In general, over the course of deliberation, jurors appear to focus more on the important facts and issues, come to a clearer understanding of them, and approach consensus on the facts." This literature also suggests the importance of the interaction between decision rules and deliberation protocols. For instance, Hastie, Penrod, and Pennington (1983) point out that the volume of discussion substantially increases with the decision rule.

Importance of deliberation and verdict patterns. In terms of the underlying assumptions of the model, there is evidence suggesting the importance of deliberation and the idea of collective information collection. Hannaford et al. (2000) studied the timing of jury opinion formation. Their data include survey responses of 1,385 jurors from 172 trials. Over 95 percent of jurors reported changing their minds at least once over the course of the trial. Importantly, over 40 percent of jurors reported changing their minds during the final deliberations, suggesting that deliberation is a key component of opinion formation for jurors.

Hans (2007) studied jury deliberation by using surveys conducted by the National Center for State Courts. The data contain reports from close to 3,500 jurors who had participated in felony trials. Figure 6 summarizes one key finding by Hans (2007). The figure groups each jury into five categories depending on the outcome of an initial straw poll. These go from "strongly favor innocent" where the great majority of jurors initially favored acquittal, to "leaning toward innocent," where a small majority initially favors acquittal, to "closely divided," where the jury is evenly split (5-7, 6-6, or 7-5), to "leaning toward guilty," and finally to "favor guilty." For each initial leaning of the jury, the figure describes the distribution of ultimate outcomes.

Note first, that the patterns of opinion change are consistent with collective information acquisition driven by a Bayesian updating process (as in our model). When the initial vote in the jury strongly supports a particular outcome, that outcome is more likely to ultimately emerge. For instance, 77 of the 89 juries with strong majorities for guilt convicted the defendant. However, the ultimate outcome does change during deliberation: 11 of these 89 juries ended up acquitting the defendant. Recall that the verdict had to be unanimous. Therefore, in all these 11 cases almost all jurors changed their mind during

by two opposing parties during the trial to figure out the relevance of different pieces of information and the weight to attribute to these in converging to a verdict. Thus, it makes sense to think of part of the deliberation process as a continuation of the information acquisition that took place during trial. 


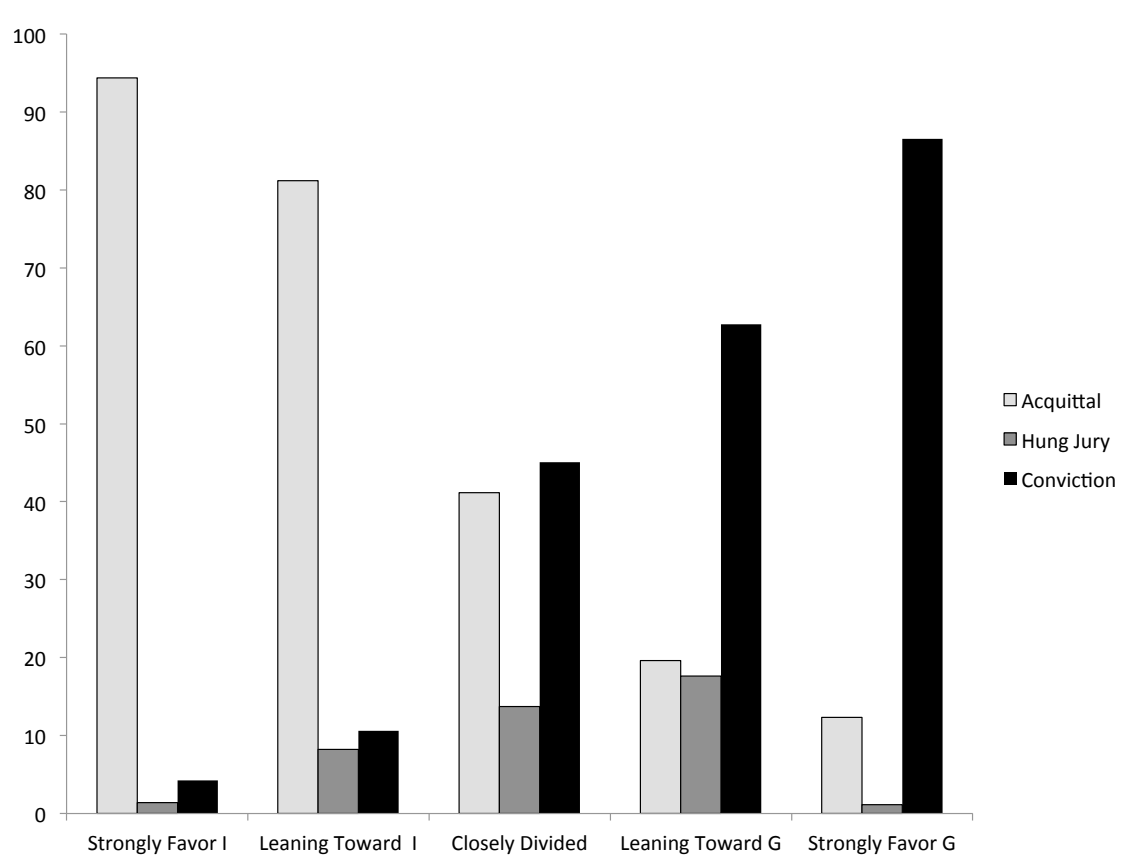

Figure 6. Distribution of jury outcomes according to initial jury leanings. Source: Hans (2007).

deliberation..$^{22}$ Thus, the deliberation process did have a large effect in these juries.

Jury composition. In line with our Proposition 4, increased heterogeneity in static preferences has been found to increase quality and length of deliberation; see Goeree and Yariv (2011), who present experimental evidence that increased preference heterogeneity increases deliberation length and accuracy of decisions. In our model, more diverse static preferences increase the length of deliberation since the pivotal members at the deliberation stage become more extreme. This translates immediately into longer deliberation and, in symmetric committees, more accurate decisions.

Effects of the decision rule. The message of our Proposition 8 is that, under some conditions, the decision rule in two-stage process does not affect outcomes. Baldwin and McConville (1980) studied a reform in 1974 in England that allowed for majority verdicts in criminal trials, while prior to the reform unanimity was required. A predominance of verdicts ( 311 out of 326 cases) were unanimous even after the reform, suggesting that the decision rule did not have much of an effect. Kalven and Zeisel (1966) report similar patterns for U.S. states that do not require unanimity for conviction: most verdicts are unanimous anyway. Hastie, Penrod, and Pennington (1983) find, however, that the volume of discussion substantially increases with the decision rule. In laboratory experiments, Goeree and Yariv (2011) find that, when subjects cannot talk before voting, the

\footnotetext{
${ }^{22}$ Initial consensus may be overstated as first poll is not taken until some consensus has already emerged.
} 
decision rule has an effect, whereas, when subjects can talk, the decision rule has very little effect. ${ }^{23}$ Our model provides a possible explanation for the fact that in some circumstances the decision rule seems to have little effect on decisions. We show that, when agents are sufficiently patient, in equilibrium, deliberation always ends with unanimous decisions: whenever there is disagreement on the appropriate decision to take, members of the committee agree that it is worthwhile to continue deliberating.

\subsection{Standard-setting Committees}

Standard-setting organizations are voluntary organizations that provide a platform for interested parties to coordinate on technological standards. Some standard-setting organizations consist solely of platform sponsors; others include also user groups, academics, and government agencies (e.g., the American National Standards Institute, or the World Wide Web Consortium). The standard-setting process begins when one or multiple sponsors submit proposed standards. A committee is then formed to consider the pros and cons of different proposals. Expert opinions play an important role in this process. For example, laboratory and field experimental studies were commissioned to evaluate different bar code designs (Brown 1997).

The evaluation process takes time. The average duration to adopt an internet standard is about 3.5 years (Simcoe 2012). In addition to different preferences over alternatives, various parties may also disagree over the timing of the decision. In the history of standardization of containers, trucking firms had lower stakes than shipping lines because the latter had to make substantial investments in ships and docks. As a result, trucking firms wanted to reach agreement on container sizes more quickly (Levinson 2006). More generally, sponsors, who have vested interests in their own technologies tend to be more patient, while users-downstream firms that build applications on the platform-may prefer quicker resolution.

There is a small literature on standard-setting committees. Farrell and Saloner (1988) and Farrell and Simcoe (2012) model the standardization process as a war of attrition between two platform sponsors. The perspective ignores the fact that deliberation, while costly, also produces valuable information. Simcoe (2012) introduces a model of collective search, whereby each sponsor draws a new value for its technology. In reality, the alternatives under consideration are not fixed, but as long as these modifications are marginal rather than radical, our model captures the strategic effects resulting from the evolving nature of the information accumulation process.

There is no consensus on what decision rules a standard-setting organization should use to adopt standards. Chiao, Lerner, and Tirole (2007) report that in their sample of standard-setting organizations, 34 percent use majority rule, 27 percent use super-

\footnotetext{
${ }^{23}$ Blinder and Morgan (2008) also find little differences in outcomes when decision rules are changed in an experiment concerning monetary policy.
} 
majority rules, and 13 percent use consensus to adopt standards. Our results suggest that there is no single decision rule that suits all standard-setting organizations. Majority rule may be more suitable to organizations whose members have similar time preferences, such as those that consist solely of technology providers, as it ensures that the adopted standard is preferred by a majority of the members. For standard-setting organizations that have a diverse membership or when the standard affects the welfare of non-members, a super-majority rule may perform better, as it promotes deliberation and prevents low-stake or impatient members from exerting disproportionate influence. Our results, however, also point out the potential danger of a high majority requirement. Instead of promoting consensus, such rules may also allow a deep-pocketed firm, who can afford a drawn-out process, to force other interested parties to concede.

\section{Conclusions}

Deliberation is an integral part of many real-life collective decisions. In addition to the examples mentioned up to now, there has been a more recent surge of interest in the idea of "deliberative democracy" in political science (e.g., Gutmann and Thompson 2004; Fishkin 2009; Steiner 2012), emphasizing consensus building more than preference revelation.

In this paper, we introduce a formal model of open-ended collective deliberations and explain how different decision rules aggregate heterogenous static and time preferences. Our analysis highlights the difference between static voting and dynamic deliberation. Whereas super-majority rules favor a particular alternative in a static environment, they force agents to gather more information in deliberative processes. We show that several features of our model are consistent with jury behavior, and our results explain why many deliberative bodies adopt super-majority rules. 


\section{Appendix}

Proof of Lemma 1. Part 1. A direct calculation gives:

$$
\frac{\partial u_{i}}{\partial G}=\frac{\left(R_{2}-R_{1}\right) e^{-(G-g)} e^{\theta} \Psi_{i}}{\left(1+e^{\theta}\right)\left(e^{-R_{1}(G-g)}-e^{-R_{2}(G-g)}\right)}\left(e^{v_{i}-g}-e^{f\left(r_{i}, G-g\right)}\right),
$$

where

$$
f\left(r_{i}, G-g\right)=\log \frac{R_{2} e^{R_{1}(G-g)}-R_{1} e^{R_{2}(G-g)}}{R_{2}-R_{1}} .
$$

Therefore, the sign of $\partial u_{i} / \partial G$ depends only on the sign of $v_{i}-g-f\left(r_{i}, G-g\right)$ and not on the value of $\theta$. Similarly,

$$
\frac{\partial u_{i}}{\partial g}=\frac{\left(R_{2}-R_{1}\right) e^{-(G-g)} e^{v_{i}} \psi_{i}}{\left(1+e^{\theta}\right)\left(e^{-R_{1}(G-g)}-e^{-R_{2}(G-g)}\right)}\left(e^{f\left(r_{i}, G-g\right)}-e^{G-v_{i}}\right) .
$$

Therefore, the sign of $\partial u_{i} / \partial g$ depends only on the sign of $f\left(r_{i}, G-g\right)-G+v_{i}$ and not on the value of $\theta$.

Part 2. The derivative $\partial u_{i} / \partial G$ has the same sign as $v_{i}-g-f\left(r_{i}, G-g\right)$, which is strictly decreasing in $G$. Similarly, $\partial u_{i} / \partial g$ has the same sign as $f\left(r_{i}, G-g\right)-G+v_{i}$, which is strictly decreasing in $g$.

Proof of Lemma 2. We only establish the statements for the upper best-response function; the proof for the corresponding part (b) follows from similar reasoning.

Part 1. From the proof of Lemma 1, the sign of $\partial u_{i} / \partial G$ depends only on the sign of $v_{i}-g-f\left(r_{i}, G-g\right)$ when $\theta \in[g, G]$. Suppose $g \geq v_{i}$. As $f\left(r_{i}, G-g\right)>0$ when $G>g$, we have $\partial u_{i} / \partial G<0$. This shows that $\Phi_{i}(g)=g$ when $g \geq v_{i}$.

Suppose $g<v_{i}$. As $v_{i}-g-f\left(r_{i}, G-g\right)$ is strictly decreasing in $G$, and is strictly negative at $G=g$ and strictly positive when $G$ is sufficiently large, there is a unique $G^{\prime}>g$ such that $\partial u_{i} / \partial G=0$ at $G=G^{\prime}$. By Lemma $1, u_{i}\left(g, G^{\prime} \mid \theta\right) \geq u(g, G \mid \theta)$ for any $G \geq g$ and for all $\theta \in\left[g, \min \left\{G, G^{\prime}\right\}\right] \cup\left[\max \left\{G, G^{\prime}\right\}, \infty\right]$. If $G<\theta<G^{\prime}$, then $u_{i}\left(g, G^{\prime} \mid \theta\right)>u_{i}(g, \theta \mid \theta)=$ $u_{i}(g, G \mid \theta)$. If $G>\theta>G^{\prime}$, then $u_{i}(g, G \mid \theta)<u_{i}(g, \theta \mid \theta)=u_{i}\left(g, G^{\prime} \mid \theta\right)$. This shows that $\Phi_{i}(g)=G^{\prime}>g$ when $g<v_{i}$.

The continuity of $\Phi_{i}$ follows from the continuity of $f\left(r_{i}, G-g\right)$ and the fact that $f\left(r_{i}, 0\right)=0$. Thus, $\lim _{g \uparrow v_{i}} \Phi_{i}(g)=v_{i}$. Twice differentiability follows from the implicit function theorem, since the function defining the first-order condition is smooth.

Part 2. To show that $\lim _{g \rightarrow-\infty} \Phi_{i}(g)=-\infty$, suppose to the contrary that there is a finite lower bound $b$ to $\Phi_{i}(g)$. We first show that there exists a $g^{\prime}$ such that $v_{i}-g-f\left(r_{i}, b-g\right)<0$ 
for all $g<g^{\prime}$. To see this, observe that

$$
\lim _{g \rightarrow-\infty} \frac{f\left(r_{i}, b-g\right)}{v_{i}-g}=\lim _{g \rightarrow-\infty} f_{2}\left(r_{i}, b-g\right)=R_{2}>1 .
$$

Furthermore, for any $g<g^{\prime}$ and $g<v_{i}, v_{i}-g-f\left(r_{i}, G-g\right)>0$ when $G=g$. Thus, there exists a $G^{\prime} \in(g, b)$ that satisfies $v_{i}-g-f\left(r_{i}, G^{\prime}-g\right)=0$. Because $\Phi_{i}(g)=G^{\prime}$ when $g<v_{i}$, it follows that $\Phi_{i}(g)<b$ for all $g<g^{\prime}$, a contradiction.

Let $f_{2}$ and $f_{22}$ represent the first and second derivatives of $f$ with respect to $G-g$. For $g<v_{i}$, differentiate the first-order condition, $\left.v_{i}-g-f\left(r_{i}, \Phi_{i}(g)-g\right)\right)=0$, with respect to $g$ to get

$$
\begin{aligned}
\Phi_{i}^{\prime}(g) & =1-\frac{1}{f_{2}} \\
\Phi_{i}^{\prime \prime}(g) & =\frac{f_{22}\left(\Phi_{i}^{\prime}-1\right)}{f_{2}^{2}} .
\end{aligned}
$$

For $G-g>0$,

$$
\begin{aligned}
f_{2} & =-R_{1} R_{2} \frac{e^{R_{2}(G-g)}-e^{R_{1}(G-g)}}{R_{2} e^{R_{1}(G-g)}-R_{1} e^{R_{2}(G-g)}}>0, \\
f_{22} & =-\frac{R_{1} R_{2}\left(R_{2}-R_{1}\right)^{2} e^{G-g}}{\left(R_{2} e^{R_{1}(G-g)}-R_{1} e^{R_{2}(G-g)}\right)^{2}}>0 .
\end{aligned}
$$

Therefore, $\Phi_{i}^{\prime}(g)<1$ and $\Phi_{i}^{\prime \prime}(g)<0$.

Finally, we show that $\Phi_{i}(g)$ reaches a maximum at $g=g_{i}^{*}$. For any $g<v_{i}$, the one-sided stopping problem satisfies a smooth pasting condition at the one-sided optimal threshold $\Phi_{i}(g)$ (see, for example, Dixit 1993). Therefore, at $G=\Phi_{i}(g)$ and $\theta=\Phi_{i}(g)$,

$$
\frac{\partial u_{i}}{\partial \theta}=\frac{\mathrm{d}\left(e^{\theta} /\left(1+e^{\theta}\right)\right)}{\mathrm{d} \theta} .
$$

Differentiate both sides with respect to $g$ to yield, at $G=\Phi_{i}(g)$ and $\theta=\Phi_{i}(g)$,

$$
\left(\frac{\mathrm{d}^{2}\left(e^{\theta} /\left(1+e^{\theta}\right)\right)}{\mathrm{d} \theta^{2}}-\frac{\partial^{2} u_{i}}{\partial \theta \partial G}-\frac{\partial^{2} u_{i}}{\partial \theta^{2}}\right) \Phi_{i}^{\prime}(g)=\frac{\partial^{2} u_{i}}{\partial \theta \partial g} .
$$

At $g=g_{i}^{*}$, we have $G=\Phi_{i}\left(g_{i}^{*}\right)=G_{i}^{*}$. Both $\partial u_{i} / \partial G$ and $\partial u_{i} / \partial g$ are equal to 0 at the optimal thresholds, and dynamic consistency implies that this value does not change with $\theta$. Therefore, at $g=g_{i}^{*}, G=G_{i}^{*}$ and $\theta=G_{i}^{*}$, we obtain:

$$
\left(\frac{\mathrm{d}^{2}\left(e^{\theta} /\left(1+e^{\theta}\right)\right)}{\mathrm{d} \theta^{2}}-\frac{\partial^{2} u_{i}}{\partial \theta^{2}}\right) \Phi_{i}^{\prime}\left(g_{i}^{*}\right)=0 .
$$


Because the stochastic process $\mathrm{d} S^{\prime}$ has an expected drift $\mu^{\prime}\left(e^{\theta}-1\right) /\left(1+e^{\theta}\right)$ and an instantaneous variance $2 \mu^{\prime}$, applying Ito's lemma gives:

$$
\frac{r}{\mu^{\prime}} u_{i}=\frac{e^{\theta}-1}{1+e^{\theta}} \frac{\partial u_{i}}{\partial \theta}+\frac{\partial^{2} u_{i}}{\partial \theta^{2}} .
$$

Using the smooth pasting condition for $\partial u_{i} / \partial \theta$, we obtain:

$$
\frac{\mathrm{d}^{2}\left(e^{\theta} /\left(1+e^{\theta}\right)\right)}{\mathrm{d} \theta^{2}}-\frac{\partial^{2} u_{i}}{\partial \theta^{2}}=-\frac{r}{\mu^{\prime}} u_{i}<0 .
$$

This establishes that $\Phi_{i}^{\prime}\left(g_{i}^{*}\right)=0$. When $g<v_{i}$, as $\Phi_{i}(g)$ is strictly concave, it reaches a maximum at $g=g_{i}^{*}$.

Part 3. For $g<v_{i}, \Phi_{i}(g)$ satisfies $v_{i}-g-f\left(r_{i}, \Phi_{i}(g)-g\right)=0$. Because the left side of this equation increases without bound in $v_{i}$, and because $f_{2}>0, \Phi_{i}(g)$ increases without bound in $v_{i}$. Moreover, for $g \leq v_{i}$, a marginal increase in $v_{i}$ has no effect on $\Phi_{i}(g)$, whereas raising $v_{i}$ to $v_{i}^{\prime}>g$ will bring $\Phi_{i}(g)$ from $g$ to a value strictly greater than $g$.

Let $f_{1}$ be the derivative of $f$ with respect to $r_{i}$. From the definitions of $R_{1}$ and $R_{2}$, we note that $\partial R_{2} / \partial r_{i}>0$ and $\partial R_{1} / \partial r_{i}=-\partial R_{2} / \partial r_{i}$. Therefore, $f_{1}$ has the same sign as:

$$
\begin{aligned}
& e^{\left(R_{2}-R_{1}\right)(G-g)}-\frac{1+\left(R_{2}-R_{1}\right) R_{2}(G-g)}{1-\left(R_{2}-R_{1}\right) R_{1}(G-g)} \\
> & 1+\left(R_{2}-R_{1}\right)(G-g)-\frac{1+\left(R_{2}-R_{1}\right) R_{2}(G-g)}{1-\left(R_{2}-R_{1}\right) R_{1}(G-g)} \\
= & \frac{-\left(R_{2}-R_{1}\right)^{2} R_{1}(G-g)^{2}}{1-\left(R_{2}-R_{1}\right) R_{1}(G-g)}>0 .
\end{aligned}
$$

Because $f\left(r_{i}, G-g\right)$ increases in both $r_{i}$ and $G-g, \Phi_{i}(g)$ decreases in $r_{i}$ for $g<v_{i}$. For $g \geq v_{i}, \Phi_{i}(g)$ does not change with $r_{i}$.

Furthermore, we have $f(0, G-g)=0$ and

$$
\lim _{r_{i} \rightarrow \infty} f\left(r_{i}, G-g\right)=\lim _{r_{i} \rightarrow \infty}\left(1+\frac{r_{i}}{\mu^{\prime}}\right)\left(e^{R_{1}(G-g)}+e^{R_{2}(G-g)}\right)=\infty .
$$

Thus, for any $g<v_{i}$ and any $\varepsilon>0$, we can choose $\bar{r}_{i}$ such that

$$
v_{i}-g-f\left(\bar{r}_{i}, \varepsilon\right)=0 .
$$

Because $f_{1}>0$ and $f_{2}>0$, for any $r_{i} \geq \bar{r}_{i}$ we must have $\Phi_{i}(g)-g \leq \varepsilon$.

We use Lemma 2 to establish two additional claims. Claim 1 is concerned with the property of the composite function $\Phi_{i}\left(\phi_{i}(G)\right)$. Claim 2 says that if the waiting region is 
narrower than the optimal waiting region for agent $i$, then he strictly prefers to adopt at least one of the alternatives later. Likewise, if the waiting region is wider than his optimal waiting region, then he prefers to adopt one of the alternatives earlier.

Claim 1. For any agent $i$,

1. $\Phi_{i}\left(\phi_{i}(G)\right)>G$ for all $G \in\left(v_{i}, G_{i}^{*}\right)$;

2. $\Phi_{i}\left(\phi_{i}(G)\right)<G$ for all $G>G_{i}^{*}$.

Proof. Part 1. Let $h(G) \equiv \Phi_{i}\left(\phi_{i}(G)\right)-G$. Then for $G \in\left(v_{i}, G_{i}^{*}\right)$,

$$
h^{\prime \prime}(G)=\Phi_{i}^{\prime \prime}\left(\phi_{i}(G)\right)\left[\phi_{i}^{\prime}(G)\right]^{2}+\Phi_{i}^{\prime}\left(\phi_{i}(G)\right) \phi_{i}^{\prime \prime}(G)<0,
$$

because by part 2 of Lemma $2, \Phi_{i}$ is strictly concave, $\phi_{i}$ is strictly convex, and $\Phi_{i}^{\prime}\left(\phi_{i}(G)\right)<$ 0 since $\phi_{i}(G)>g_{i}^{*}$. Thus, $h$ is strictly concave for $G \in\left(v_{i}, G_{i}^{*}\right)$. This, together with $h\left(v_{i}\right)=h\left(G_{i}^{*}\right)=0$, implies that $h(G)>0$ for $G \in\left(v_{i}, G_{i}^{*}\right)$.

Part 2. Since $\max _{g} \Phi_{i}(g)=G_{i}^{*}$, we have $h(G) \leq G_{i}^{*}-G<0$ for all $G>G_{i}^{*}$.

Claim 2. For any agent $i$ and any $(g, G) \notin\left\{\left(g_{i}^{*}, G_{i}^{*}\right),\left(v_{i}, v_{i}\right)\right\}$,

1. if $G-g \leq G_{i}^{*}-g_{i}^{*}$, then either $g>\phi_{i}(G)$ or $G<\Phi_{i}(g)$;

2. if $G-g \geq G_{i}^{*}-g_{i}^{*}$, then either $g<\phi_{i}(G)$ or $G>\Phi_{i}(g)$.

Proof. Part 1. Suppose $G-g \leq G_{i}^{*}-g_{i}^{*}$. (i) If $G>G_{i}^{*}$, then because $G-\phi_{i}(G)$ is strictly increasing in $G$ (part 2 of Lemma 2), we have $G-\phi_{i}(G)>G_{i}^{*}-\phi_{i}\left(G_{i}^{*}\right) \geq G-g$. Hence, $g>\phi_{i}(G)$. (ii) If $g<g_{i}^{*}$, then because $\Phi_{i}(g)-g$ is strictly decreasing in $g$, we have $\Phi_{i}(g)-g>\Phi_{i}\left(g_{i}^{*}\right)-g_{i}^{*} \geq G-g$. Hence, $\Phi_{i}(g)>G$. (iii) Suppose $g \geq g_{i}^{*}$ and $G \leq G_{i}^{*}$. If $G>g \geq v_{i}$, then $\phi_{i}(G)<v_{i} \leq g$ because $\phi_{i}$ is decreasing for $G \in\left[v_{i}, G_{i}^{*}\right]$. If $v_{i} \geq G>g$, then $\Phi_{i}(g)>v_{i} \geq G$ because $\Phi_{i}$ is decreasing for $g \in\left[g_{i}^{*}, v_{i}\right]$. If $G \geq v_{i} \geq g$, then by Claim $1, \Phi_{i}\left(\phi_{i}(G)\right)>G$ for $G \in\left(v_{i}, G_{i}^{*}\right)$. As $\Phi_{i}$ is decreasing for $g \in\left[g_{i}^{*}, v_{i}\right]$, if $g \leq \phi_{i}(G)$ then we must have $\Phi_{i}(g) \geq \Phi_{i}\left(\phi_{i}(G)\right)>G_{i}$.

Part 2. Suppose $G-g \geq G_{i}^{*}-g_{i}^{*}$. Because $(g, G) \neq\left(g_{i}^{*}, G_{i}^{*}\right)$, either $G>G_{i}^{*}$ or $g<g_{i}^{*}$. If $G>G_{i}^{*}$, then $G>\Phi_{i}(g)$ for any $g$. If $g<g_{i}^{*}$, then $g<\phi_{i}(G)$ for any $G$.

Proof of Lemma 3. A cutoff pair $(\hat{g}, \hat{G})$, with $\hat{g} \neq \hat{G}$, solves the constrained optimization problem for some $\theta$ within the boundaries only if it satisfies the Kuhn-Tucker conditions:

$$
\frac{\partial u_{i}}{\partial G}\left\{\begin{array} { l l } 
{ \leq 0 } & { \text { if } \hat { G } = \underline { G } _ { i } , } \\
{ = 0 } & { \text { if } \hat { G } \in ( \underline { G } _ { i } , \overline { G } _ { i } ) , } \\
{ \geq 0 } & { \text { if } \hat { G } = \overline { G } _ { i } ; }
\end{array} \quad \frac { \partial u _ { i } } { \partial g } \left\{\begin{array}{ll}
\leq 0 & \text { if } \hat{g}=\underline{g}_{i}, \\
=0 & \text { if } \hat{g} \in\left(\underline{g}_{i}, \bar{g}_{i}\right), \\
\geq 0 & \text { if } \hat{g}=\bar{g}_{i} .
\end{array}\right.\right.
$$


From the definition of the the best-response functions, $(\hat{g}, \hat{G})$ satisfies the Kuhn-Tucker conditions if and only if it satisfies (2) and (3).

We now show that there is only one pair of thresholds that satisfies the Kuhn-Tucker conditions. Suppose, by way of contradiction, that $\left(g^{\prime}, G^{\prime}\right)$ and $(\hat{g}, \hat{G})$ both satisfy the Kuhn-Tucker conditions. Because the one-sided preferences are single-peaked, $g^{\prime} \neq \hat{g}$ and $G^{\prime} \neq \hat{G}$. There are two cases to consider.

Case 1: $g^{\prime}>\hat{g}$ and $G^{\prime}>\hat{G}$. Because $g^{\prime}>\underline{g}_{i}$ and $G^{\prime}>\underline{G}_{i}$, the Kuhn-Tucker conditions require that at the point $\left(g^{\prime}, G^{\prime}\right), \partial u_{i} / \partial g \geq 0$ and $\partial u_{i} / \partial G \geq 0$. (This means that $\left(g^{\prime}, G^{\prime}\right)$ is in region $Q 2$ of Figure 1.) Therefore, $g^{\prime} \leq \phi_{i}\left(G^{\prime}\right)$ and $G^{\prime} \leq \Phi_{i}\left(g^{\prime}\right)$. Because $\Phi_{i}\left(g^{\prime}\right) \leq G_{i}^{*}$, $\hat{G}<G^{\prime} \leq \Phi_{i}\left(g^{\prime}\right) \leq G_{i}^{*}$. By part 2 of Lemma $2, \phi_{i}(\hat{G})>\phi_{i}\left(G^{\prime}\right) \geq g^{\prime}>\hat{g}$. Hence, $\partial u_{i} / \partial g>0$ at $(\hat{g}, \hat{G})$. (Intuitively, because $(\hat{g}, \hat{G})$ is south-west of $\left(g^{\prime}, G^{\prime}\right)$, it must belong to either region $Q 1$ or $Q 2$ in Figure 1.) However, because $\hat{g}<\bar{g}_{i}$, the Kuhn-Tucker conditions require $\partial u_{i} / \partial g \leq 0$ at that point.

Case 2: $g^{\prime}>\hat{g}$ and $G^{\prime}<\hat{G}$. Because $g^{\prime}>\underline{g}_{i}$ and $G^{\prime}<\bar{G}_{i}$, the Kuhn-Tucker conditions require that at the point $\left(g^{\prime}, G^{\prime}\right), \partial u_{i} / \partial g \geq 0$ and $\partial u_{i} / \partial G \leq 0$. (This means that $\left(g^{\prime}, G^{\prime}\right)$ is in region $Q 1$ in Figure 1.) By part 1 of Claim $2, G^{\prime}-g^{\prime}>G_{i}^{*}-g_{i}^{*}$. Because $\hat{G}-\hat{g}>G^{\prime}-g^{\prime}$, $\hat{G}-\hat{g}>G_{i}^{*}-g_{i}^{*}$. It then follows from part 2 of Claim 2 that either $\partial u_{i} / \partial g>0$ or $\partial u_{i} / \partial G<0$ at $(\hat{g}, \hat{G})$. (Intuitively, as $\Phi_{i}$ has a positive slope for $g \leq g_{i}^{*}$ and $\phi_{i}$ has a positive slope for $G \geq G_{i}^{*},(\hat{g}, \hat{G})$, which is north-west of $\left(g^{\prime}, G^{\prime}\right)$, must belong to region $Q 1$.) However, as $\hat{g}<\bar{g}_{i}$ and $\hat{G}>\underline{G}_{i}$, the Kuhn-Tucker conditions require $\partial u_{i} / \partial g \leq 0$ and $\partial u_{i} / \partial G \geq 0$ at that point.

Thus, for any $\theta$, the only $(g, G)$ that satisfies the Kuhn-Tucker conditions with $\theta \in$ $(g, G)$ is the pair $(\hat{g}, \hat{G})$. Because a solution to the constrained maximization problem (1) always exists, $(\hat{g}, \hat{G})$ is a solution to (1) if $u_{i}(\hat{g}, \hat{G} \mid \theta)$ is greater than $e^{v_{i}} /\left(1+e^{\theta}\right)$ when adopting $\beta$ immediately is feasible at $\theta$, and greater than $e^{\theta} /\left(1+e^{\theta}\right)$ when adopting $\alpha$ immediately is feasible.

Suppose $\theta \in[\hat{g}, \hat{G}]$. Adopting $\beta$ immediately is feasible if $\theta<\bar{g}_{i}$. In this case, the KuhnTucker conditions imply that at the point $(\hat{g}, \hat{G} ; \theta), \partial u_{i} / \partial g \leq 0$ (as $\left.\hat{g} \leq \theta<\bar{g}_{i}\right)$. It follows from Lemma 1 that $u_{i}(\hat{g}, \hat{G} \mid \theta) \geq u_{i}(\theta, \hat{G} \mid \theta)=e^{v_{i}} /\left(1+e^{\theta}\right)$. Adopting $\alpha$ immediately is feasible if $\theta \geq \underline{G}_{i}$. In this case, the Kuhn-Tucker conditions imply that at the point $(\hat{g}, \hat{G} ; \theta)$, $\partial u_{i} / \partial G \geq 0$, and it follows that $u_{i}(\hat{g}, \hat{G} \mid \theta) \geq u_{i}(\hat{g}, \theta \mid \theta)=e^{\theta} /\left(1+e^{\theta}\right)$.

Suppose $\theta>\hat{G}$. Under $(\hat{g}, \hat{G}), \alpha$ is adopted immediately. If adopting $\beta$ immediately is feasible (i.e., $\theta<\bar{g}_{i}$ ), then it must also be feasible at $\hat{G}$. But we have already shown in the last paragraph that agent $i$ prefers $\alpha$ to $\beta$ at $\hat{G}$ whenever adopting $\beta$ immediately is feasible. Because the relative payoff of $\alpha$ increases an agent's belief, agent $i$ must also prefer $\alpha$ to $\beta$ at $\theta$. By similar logic, if $\alpha$ is feasible at some $\theta<\hat{g}$, agent $i$ must prefer $\beta$ to $\alpha$. 
Proof of Proposition 1. Necessary and sufficient condition for equilibrium. Sufficiency. Suppose $(\hat{g}, \hat{G})$ is an unequal fixed point of $\left(\phi_{p i v}, \Phi_{p i v}\right)$. Consider the strategy profile $\sigma$ where, for each $i$,

$$
\sigma_{i}=\left(\phi_{i}(\hat{G}), \Phi_{i}(\hat{g})\right) .
$$

It is straightforward to see that $\left(g^{[k]}(\sigma), G^{[k]}(\sigma)\right)=(\hat{g}, \hat{G})$. Furthermore, for each agent $i, \hat{g}$ satisfies (2) given $\hat{G}$, and $\hat{G}$ satisfies (3) given $\hat{G}$. Hence, by Lemma 3, $\sigma$ is a Nash equilibrium. Furthermore, by the single-crossing property of Lemma 1, $\sigma$ also satisfies condition 2 of the equilibrium definition. The above argument shows that if $(\hat{g}, \hat{G})$ is an unequal fixed point of $\left(\phi_{p i v}, \Phi_{p i v}\right)$, then it is an equilibrium outcome of the game.

Necessity. Suppose $\sigma$ is an equilibrium strategy profile. Let $(\hat{g}, \hat{G})=\left(g^{[k]}(\sigma), G^{[k]}(\sigma)\right)$. By the single-crossing property of Lemma 1, we can restate condition 2 of Definition 1 as requiring that, for each agent $i, G_{i}>\hat{G}$ if $\Phi_{i}(\hat{g})>\hat{G}$ and $G_{i}<\hat{G}$ if $\Phi_{i}(\hat{g})<\hat{G}$. It follows that a pivotal agent $i$ with $G_{i}=\hat{G}$ must have $G_{i}=\Phi_{i}(\hat{g})$. There are $k-1$ agents with $G_{j} \leq \hat{G}$; the lower bound of their upper spans of control $I^{G}\left(\sigma_{-j}\right)$ is $\hat{G}$ because they can delay but not accelerate the adoption of $\alpha$. By Lemma 3 we have $\Phi_{j}(\hat{g}) \leq \hat{G}$ for each of these $k-1$ agents. Similarly, there are $2 m-k-1$ agents with $G_{j} \geq \hat{G}$; the upper bound of their upper spans of control $I^{G}\left(\sigma_{-j}\right)$ is $\hat{G}$ because they can accelerate but not delay the adoption of $\alpha$. By Lemma 3 we have $\Phi_{j}(\hat{g}) \geq \hat{G}$ for these agents. This argument establishes that agent $i$ 's upper best-response function is the $k$-th smallest upper bestresponse function when the lower cutoff is at $\hat{g}$. Hence, $\hat{G}=\Phi_{i}(\hat{g})=\Phi_{p i v}(\hat{g})$. Similar reasoning for the lower best-response shows that $\hat{g}=\phi_{\text {piv }}(\hat{G})$. Thus, $(\hat{g}, \hat{G})$ must be a fixed point of $\left(\phi_{p i v}, \Phi_{p i v}\right)$.

Non-degeneracy. We now show that $g^{[k]}(\sigma)<G^{[k]}(\sigma)$. Note that $(x, x)$ is an equal fixed point of $\left(\phi_{p i v}, \Phi_{p i v}\right)$ if $\phi_{p i v}(x)=\Phi_{p i v}(x)=x$. When $g \geq v_{k}, \Phi_{i}(g)=g$ for $i \leq k$ and $\Phi_{i}(g)>g$ for $i>k$. Hence, $\Phi_{p i v}(g)=g$ for $g \geq v_{k}$. In contrast, when $g<v_{k}, \Phi_{i}(g)>g$ for all $i \geq k$. It follows that $\Phi_{\text {piv }}(g)>g$ for $g<v_{k}$. By similar logic, $\phi_{p i v}(G)=G$ when $G \leq v_{2 m-k}$ and $\phi_{p i v}(G)<G$ when $G>v_{2 m-k}$. Because $v_{2 m-k}>v_{k}$ when $k>m,\left(\phi_{p i v}, \Phi_{p i v}\right)$ has no equal fixed point when $k>m$.

When $k=m$, the only equal fixed point is $\left(v_{m}, v_{m}\right)$. Suppose, by way of contradiction, that $\left(v_{m}, v_{m}\right)$ is an equilibrium outcome. Condition 2 of Definition 1 would require each $i>m$ to choose $G_{i}>v_{m}$ and each $j<m$ to choose $g_{j}<m$. If agent $m$ chooses $G_{m}>v_{m}$, then $\alpha$ will not be adopted at $v_{m}$. In other words, agent $m$ can delay the adoption of $\alpha$ by choosing $G_{m}>v_{m}$. By a similar logic, agent $m$ can delay the adoption of $\beta$ by choosing $g_{m}<v_{m}$. Pick $\varepsilon \in\left(0, G_{m}^{*}-v_{m}\right)$ such that agent $m$ can delay the adoption of $\alpha$ by $\varepsilon$ and the adoption of $\beta$ by $v_{m}-\phi_{m}\left(v_{m}+\varepsilon\right)$. Because agent $m$ 's span of control is non-degenerate, such deviation is feasible if $\varepsilon$ is sufficiently small. From Claim $1, \Phi_{m}\left(\phi_{m}\left(v_{m}+\varepsilon\right)\right)>v_{m}+\varepsilon$. Hence, by Lemma 1,

$$
u_{m}\left(\phi_{i}\left(v_{m}+\varepsilon\right), v_{m}+\varepsilon \mid v_{m}\right)>u_{m}\left(\phi_{i}\left(v_{m}+\varepsilon\right), v_{m} \mid v_{m}\right)=e^{v_{m}} /\left(1+e^{v_{m}}\right) .
$$


So $\left(v_{m}, v_{m}\right)$ cannot be optimal for agent $m$.

Equilibrium existence. An equilibrium exists if and only if $\left(\phi_{p i v}, \Phi_{p i v}\right)$ has an unequal fixed point. Consider any $k \geq m$. Given that for any $x<v_{1}$ and all $i, \phi_{i}(x)=x$ and $\Phi_{i}(x)>x$, we have $\Phi_{p i v}\left(\phi_{p i v}(x)\right)>x$. Because for all $i, \Phi_{i}(x) \leq G_{i}^{*}$, we have $\Phi_{\text {piv }}\left(\phi_{\text {piv }}(x)\right)<x$ for any $x>\max _{i}\left\{G_{i}^{*}\right\}$. By continuity, there exists $x^{\prime} \in\left[v_{1}, \max _{i}\left\{G_{i}^{*}\right\}\right]$, such that $\Phi_{\text {piv }}\left(\phi_{\text {piv }}\left(x^{\prime}\right)\right)=x^{\prime}$. Hence, $\left(\phi_{p i v}, \Phi_{\text {piv }}\right)$ has a fixed point under any rule $k \geq m$.

We have already shown that $\left(\phi_{p i v}, \Phi_{p i v}\right)$ has no equal fixed point under a super-majority rule $k>m$. Hence, any fixed point under rule $k>m$ must be unequal. Finally, consider majority rule $k=m$. Note that

$$
\begin{array}{ll}
\Phi_{i}(g)=g & \text { for } g \geq v_{m-1} \text { and } i<m \\
\Phi_{i}(g)>g & \text { for } g<v_{m+1} \text { and } i>m .
\end{array}
$$

Because $\Phi_{m}$ is continuous, $\Phi_{m}\left(v_{m}\right)=v_{m}$, and $\Phi_{m}(g)>g$ for $g<v_{m}$, we have $\Phi_{p i v}(g)=$ $\Phi_{m}(g)$ for $g$ slightly less than $v_{m}$. By similar logic, $\phi_{p i v}(G)=\phi_{m}(G)$ for $G$ slightly greater than $v_{m}$. Hence, by Claim 1 , for $G$ slightly greater than $v_{m}$,

$$
\Phi_{\text {piv }}\left(\phi_{\text {piv }}(G)\right)=\Phi_{m}\left(\phi_{m}(G)\right)>G .
$$

It follows that under rule $k=m, \Phi_{p i v}\left(\phi_{\text {piv }}\left(G^{\prime}\right)\right)=G^{\prime}$ for some $G^{\prime} \in\left(G, \max _{i}\left\{G_{i}^{*}\right\}\right]$.

Nested equilibria. For any equilibrium outcome $(\hat{g}, \hat{G})$,

$$
\hat{G}-\phi_{p i v}(\hat{G})=\hat{G}-\hat{g}=\Phi_{p i v}(\hat{g})-\hat{g} .
$$

Suppose $\left(\hat{g}^{\prime}, \hat{G}^{\prime}\right)$ is another equilibrium outcome, and assume without loss of generality that $\hat{G}-\hat{g} \geq \hat{G}^{\prime}-\hat{g}^{\prime}$. Because $G-\phi_{p i v}(G)$ is strictly increasing in $G$ and $\Phi_{p i v}(g)-g$ is strictly decreasing in $g$ (as both $\Phi_{i}$ and $\phi_{i}$ have a slope strictly less than 1 for all $i$ ), we must have $\hat{G}>\hat{G}^{\prime}$ and $\hat{g}<\hat{g}^{\prime}$, which contradicts the initial assumption.

Equilibrium uniqueness. Unanimity. Because any agent can delay a decision under the unanimity rule, $(\hat{g}, \hat{G})$ is an equilibrium only if for all $i$

$$
\hat{g} \leq \phi_{i}(\hat{G}) ; \quad \text { and } \hat{G} \geq \Phi_{i}(\hat{g}) .
$$

By part 1 of Claim 2, we obtain $\hat{G}-\hat{g} \geq G_{i}^{*}-g_{i}^{*}$ and, hence, either $\hat{G} \geq G_{i}^{*}$ or $\hat{g} \leq g_{i}^{*}$. Suppose $\hat{G} \geq G_{i}^{*}$. Consider any waiting region $\left(g^{\prime}, G^{\prime}\right)$ that strictly contains $(\hat{g}, \hat{G})$. By part 2 of Lemma 2, $G_{i}^{*}=\max _{g} \Phi_{i}(g)$ and $\phi_{i}$ is increasing for $G>G_{i}^{*}$. Hence,

$$
\begin{aligned}
& G^{\prime}>\hat{G} \geq G_{i}^{*} \geq \Phi_{i}\left(g^{\prime}\right) ; \\
& g^{\prime}<\hat{g} \leq \phi_{i}(\hat{G})<\phi_{i}\left(G^{\prime}\right) .
\end{aligned}
$$


In other words, agent $i$ strictly prefers adopting $\alpha$ before $G^{\prime}$ and $\beta$ before $g^{\prime}$. By similar logic, the same is true when $\hat{g} \leq g_{i}^{*}$. Because given $g^{\prime}$ every agent $i$ strictly prefers to adopt $\alpha$ before $G^{\prime},\left(g^{\prime}, G^{\prime}\right)$ cannot be an equilibrium. As all equilibria must be nested, $(\hat{g}, \hat{G})$ is unique.

Homogeneous time preference. When all agents have the same discount rate, their one-sided best response functions do not cross. In this case, $\phi_{\text {piv }}(\cdot)=\phi_{2 m-k}(\cdot)$ and $\Phi_{\text {piv }}(\cdot)=$ $\Phi_{k}(\cdot)$. To prove uniqueness, we need to show that $\left(\phi_{m-k}, \Phi_{k}\right)$ has a unique unequal fixed point. For majority rule $k=m$, it follows from Claim 1 that $\left(\phi_{m}, \Phi_{m}\right)$ has a unique unequal fixed point at $\left(g_{m}^{*}, G_{m}^{*}\right)$. Consider super-majority rule $k>m$. By Claim 1 and part 3 of Lemma 2, if $G<G_{2 m-k}^{*}$ we have

$$
\Phi_{k}\left(\phi_{2 m-k}(G)\right)>\Phi_{2 m-k}\left(\phi_{2 m-k}(G)\right) \geq G .
$$

Because $\Phi_{k}(g) \leq G_{k}^{*}$ for any $g$, if $G>G_{k}^{*}$ we have

$$
\Phi_{k}\left(\phi_{2 m-k}(G)\right)<G .
$$

Given that both $\phi_{2 m-k}^{\prime}(G)$ and $\Phi_{k}^{\prime}\left(\phi_{2 m-k}(G)\right)$ are strictly positive and less than 1 for $G \in$ $\left(G_{2 m-k}^{*}, G_{k}^{*}\right), \Phi_{k}\left(\phi_{2 m-k}(G)\right)-G$ is strictly decreasing in that range. Hence, there exists a unique $G^{\prime} \in\left[G_{2 m-k}^{*}, G_{k}^{*}\right]$, such that $\Phi_{k}\left(\phi_{2 m-k}\left(G^{\prime}\right)\right)=G^{\prime}$.

Homogeneous static preference. When all agents have the same $v_{i}$, the pivotal bestresponse functions are given by the best-response functions of the agent with the $k$ th most impatient agent. Uniqueness follows Claim 1, which establishes that the bestresponse functions of any single agent has a unique unequal fixed point at the Waldoptimal thresholds.

Proof of Proposition 2. Let $i$ be an impatient agent. Assume that $i \leq m$. The case in which $i \geq m$ can be treated symmetrically. Pick any $\varepsilon \leq\left(0, v_{m+1}-v_{m}\right)$. By part 3 of Lemma 2 there exists $\bar{r}(\varepsilon)$ such that for any $r_{i} \geq \bar{r}(\varepsilon)$ and $G \leq v_{m+1}$, we have $\phi_{i}(G)>G-\varepsilon$.

For all agents $j \geq m+1, \phi_{j}(G)=G$ if $G<v_{m+1}$. Thus, $\phi_{p i v}(G) \geq \phi_{i}(G)$ if $G<v_{m+1}$. Given that $v_{m}+\varepsilon<v_{m+1}$, we have

$$
\phi_{p i v}\left(v_{m}+\varepsilon\right) \geq \phi_{i}\left(v_{m}+\varepsilon\right)>v_{m}+\varepsilon-\varepsilon=v_{m} .
$$

For all agents $j \leq m, \Phi_{j}(g)=g$ if $g>v_{m}$. Thus, $\Phi_{p i v}(g)=g$ if $g>v_{m}$. This, together with the displayed inequality above, implies that

$$
\Phi_{\text {piv }}\left(\phi_{\text {piv }}\left(v_{m}+\varepsilon\right)\right)=\phi_{\text {piv }}\left(v_{m}+\varepsilon\right)<v_{m}+\varepsilon,
$$

where the last inequality follows because $\phi_{p i v}(G)<G$ for any $G>v_{m}$. 
We have already shown in the proof of equilibrium existence in Proposition 1 that $\Phi_{\text {piv }}\left(\phi_{\text {piv }}\left(G^{\prime}\right)\right)>G^{\prime}$ for $G^{\prime}$ slightly above $v_{m}$. Therefore, there is an upper threshold $\hat{G} \in$ $\left(G^{\prime}, v_{m}+\varepsilon\right)$ that satisfies the equilibrium condition. Furthermore, because $\hat{G}<v_{m+1}$, $\phi_{\text {piv }}(\hat{G}) \geq \phi_{i}(\hat{G})$. Therefore, $\hat{G}-\phi_{\text {piv }}(\hat{G}) \leq \hat{G}-\phi_{i}(\hat{G})<\varepsilon$. The equilibrium waiting region is no wider than $\varepsilon$.

Finally, the fact that $v_{m} \in(\hat{g}, \hat{G})$ is a general property of any equilibrium under majority rule; see the proof of Proposition 6.

Proof of Proposition 3. Because $\left(g_{i}^{*}, G_{i}^{*}\right)$ is the unique unequal fixed point of $\left(\phi_{i}, \Phi_{i}\right)$, by part 1 of Proposition 4 below, $G_{i}^{*}-g_{i}^{*}$ decreases in $r_{i}$. Therefore, there exists $\tilde{r}(\varepsilon)$ such that $G_{i}^{*}-g_{i}^{*} \geq \varepsilon$ for any agent $i$ with $r_{i} \leq \tilde{r}(\varepsilon)$.

Suppose the equilibrium waiting region is $(\hat{g}, \hat{G})$ and the number of agents with $r_{i} \geq$ $\tilde{r}(\varepsilon)$ is strictly less than the number of requisite swing votes under rule $k$. Then, there must be at least one agent with $r_{i}<\tilde{r}(\varepsilon)$ who both votes for $\alpha$ at $\hat{G}$ and votes for $\beta$ at $\hat{g}$. Suppose by way of contradiction that $\hat{G}-\hat{g}<\varepsilon$. Then, for an agent with $r_{i}<\tilde{r}(\varepsilon)$, we have $\hat{G}-\hat{g}<\varepsilon \leq G_{i}^{*}-g_{i}^{*}$. But for such $(\hat{g}, \hat{G})$, by part 1 of Claim 2, either $\partial u_{i} / \partial g<0$ or $\partial u_{i} / \partial G>0$. This agent cannot both vote for $\alpha$ at $\hat{G}$ and vote for $\beta$ at $\hat{g}$, a contradiction.

Proof of Proposition 4. Part 1. We show that the width of the waiting region in the most and least patient equilibria increases whenever $\Phi_{p i v}$ shifts up or $\phi_{p i v}$ shifts down.

Let $(\hat{g}, \hat{G})$ be the fixed point of $\left(\phi_{p i v}, \Phi_{p i v}\right)$ in the most patient equilibrium. Let $\left(g^{\prime}, G^{\prime}\right)$ be the fixed point of $\left(\phi_{p i v}, \tilde{\Phi}_{p i v}\right)$ in the most patient equilibrium, where $\tilde{\Phi}_{p i v}(g) \geq \Phi_{p i v}(g)$ for all $g$. We have

$$
\tilde{\Phi}_{p i v}\left(\phi_{p i v}(\hat{G})\right)-\hat{G} \geq \Phi_{p i v}\left(\phi_{p i v}(\hat{G})\right)-\hat{G}=0 .
$$

In the proof of equilibrium existence in Proposition 1, we have shown that $\tilde{\Phi}_{\text {piv }}\left(\phi_{\text {piv }}(G)\right)$ $G<0$ for $G$ that is sufficiently large. Because multiple equilibrium thresholds are nested, $G^{\prime}$ is the largest solution to $\tilde{\Phi}_{p i v}\left(\phi_{p i v}(G)\right)-G=0$. It follows that $G^{\prime} \geq \hat{G}$. Furthermore, $G-\phi_{\text {piv }}(G)$ is increasing in $G$ because the slope of $\phi_{i}(G)$ is less than 1 for every $i$. Therefore,

$$
G^{\prime}-g^{\prime}=G^{\prime}-\phi_{p i v}\left(G^{\prime}\right) \geq \hat{G}-\phi_{p i v}(\hat{G})=\hat{G}-\hat{g} .
$$

Similar reasoning applies to the least patient equilibrium and to the case when $\phi_{p i v}$ shifts down.

Because $\Phi_{p i v}\left(g ; k^{\prime}\right) \geq \Phi_{p i v}(g ; k)$ and $\phi_{p i v}\left(G ; k^{\prime}\right) \leq \phi_{p i v}(G ; k)$ for $k^{\prime}>k$, the width of the equilibrium waiting region is increasing in $k$ both in the most patient equilibrium and in the least patient equilibrium. Similarly, a decrease in $r_{i}$ shifts up $\Phi_{i}$ and shifts down $\phi_{i}$. As a result, $\Phi_{p i v}$ also shifts up and $\phi_{p i v}$ shifts down (weakly). The waiting region in the most patient and the least patient equilibria becomes wider.

Part 2. Let $\mathcal{C}$ denote a subset of $k$ agents. The upper and lower pivotal functions for 
the group $\mathcal{C}$ under the unanimity rule are $\Phi_{*}(g) \equiv \max _{i \in \mathcal{C}} \Phi_{i}(g)$ and $\phi_{*}(G) \equiv \min _{i \in \mathcal{C}} \phi_{i}(G)$, respectively. Let $\left(g_{*}, G_{*}\right)$ denote the unique unequal fixed point of $\left(\phi_{*}, \Phi_{*}\right)$.

In the original game with $2 m-1$ agents, $\Phi_{p i v}(g) \leq \Phi_{*}(g)$ for all $g$ and $\phi_{p i v}(G) \geq \phi_{*}(G)$ for all $G$ under rule $k$, regardless of the preferences of the agents outside $\mathcal{C}$. Recall that the width of the waiting region in the most patient equilibrium increases whenever $\Phi_{\text {piv }}$ shifts up or $\phi_{\text {piv }}$ shifts down. Because $\left(g_{*}, G_{*}\right)$ is the unique, and hence most patient, fixed point of $\left(\phi_{*}, \Phi_{*}\right)$, we have

$$
\hat{G}-\hat{g} \leq G_{*}-g_{*},
$$

for any fixed point $(\hat{g}, \hat{G})$ of $\left(\phi_{p i v}, \Phi_{p i v}\right)$.

Part 3. An increase in $k$, a decrease in the common discount rate $r$, or more diverse preferences in a symmetric jury shifts up $\Phi_{p i v}$ and shifts down $\phi_{p i v}$. Thus, $\hat{G}-\hat{g}$ increases. From the proof of Lemma 1, the equilibrium cutoffs must satisfy these first-order conditions:

$$
\begin{aligned}
v_{k}-\hat{g}-f(r, \hat{G}-\hat{g}) & =0, \\
f(r, \hat{G}-\hat{g})-\hat{G}+v_{2 m-k} & =0 .
\end{aligned}
$$

Adding these two equations implies that $\hat{g}+\hat{G}=v_{k}+v_{2 m-k}=2 v_{m}$, which is fixed. Thus, $\hat{G}$ must increase and $\hat{g}$ must fall.

Proof of Proposition 5. From the proof of part 3 of Proposition 4 for a symmetric committee, the equilibrium waiting region under super-majority rule $k>m,(\hat{g}, \hat{G})$, strictly contains the equilibrium waiting region under majority rule $m,\left(g_{m}^{*}, G_{m}^{*}\right)$. Further, both waiting regions are centered at $v_{m}$.

Consider any $\theta_{0} \in\left[g_{m}^{*}, G_{m}^{*}\right]$. Instead of writing the utility of agent $j$ as a function of the cutoffs $g$ and $G$, we can define $c=(g+G) / 2$ and $y=G-g$ to express utility as a function of the center and the width of the waiting region. Using the formula in the text, and after some re-arrangement, we obtain:

$$
u_{j}\left(g, G \mid \theta_{0}\right)=\frac{1}{1+e^{\theta_{0}}} \frac{\tilde{q}_{j}\left(c, y ; \theta_{0}\right)}{q(y)} ;
$$

where

$$
\begin{aligned}
\tilde{q}_{j}\left(c, y ; \theta_{0}\right) & =\frac{\left(e^{v_{j}} e^{R_{1}\left(\theta_{0}-c\right)}+e^{c} e^{R_{2}\left(\theta_{0}-c\right)}\right) e^{-R_{1} y / 2}-\left(e^{c} e^{R_{1}\left(\theta_{0}-c\right)}+e^{v_{j}} e^{R_{2}\left(\theta_{0}-c\right)}\right) e^{-R_{2} y / 2}}{e^{-R_{1} y / 2}-e^{-R_{2} y / 2}}, \\
q(y) & =e^{-R_{1} y / 2}+e^{-R_{2} y / 2}
\end{aligned}
$$

Note that $c=v_{m}$ under both majority rule and super-majority rule. Moreover, the width of the equilibrium waiting region under majority rule, denoted $y^{*}$, is smaller than that 
under super-majority rule, denoted $\hat{y}$. At $\theta_{0}=v_{m}$, the utility of agent $j$ is

$$
u_{j}\left(g, G \mid v_{m}\right)=\frac{1}{1+e^{v_{m}}} \frac{e^{v_{j}}+e^{v_{m}}}{q(y)} .
$$

The function $q(y)$ is decreasing for $y<y^{*}$ and increasing for $y>y^{*}$. Since $\hat{y}>y^{*}$, we have $u_{j}\left(g^{*}, G^{*} \mid v_{m}\right)>u_{j}\left(\hat{g}, \hat{G} \mid v_{m}\right)$ for all $j$. By continuity, there is a region containing $v_{m}$ such that $u_{j}\left(g^{*}, G^{*} \mid \theta_{0}\right)>u_{j}\left(\hat{g}, \hat{G} \mid \theta_{0}\right)$ for all $j$ and all $\theta_{0}$ in this region.

Proof of Proposition 6. From Lemma 2, part 1, and from the definition of the pivotal bestresponse functions,

$$
\Phi_{p i v}(g ; k)\left\{\begin{array} { l l } 
{ > g } & { \text { if } g < v _ { k } , } \\
{ = g } & { \text { if } g \geq v _ { k } ; }
\end{array} \quad \phi _ { p i v } ( G ; k ) \left\{\begin{array}{ll}
<G & \text { if } G>v_{2 m-k}, \\
=G & \text { if } G \leq v_{2 m-k} .
\end{array}\right.\right.
$$

Because an equilibrium pair of thresholds $(\hat{g}, \hat{G})$ is an unequal fixed point, we must have $\hat{G}=\Phi_{p i v}(\hat{g})>\hat{g}$. This implies that $\hat{g}<v_{k}$, which means that if $\beta$ is adopted when the belief hits $\hat{g}$, at least $2 m-k$ agents (including agents $k$ to $2 m-1$ ) must prefer $\beta$ to $\alpha$ at the current belief. Similarly, the upper threshold must satisfy $\hat{G}>v_{2 m-k}$, which implies that at least $2 m-k$ agents (including agents 1 to $2 m-k$ ) must prefer $\alpha$ to $\beta$ when $\alpha$ is adopted. For $k=m$, we must have $v_{m} \in(\hat{g}, \hat{G})$, which implies that equilibrium outcomes respect the static preferences for a majority of voters.

Fix the discount rates of all agents and fix the static preferences of $k-1$ agents. We prove that there exists $\underline{v}$ such that if $v_{i} \leq \underline{v}$ for $i \leq 2 m-k$, then $\hat{G}<v_{2 m-k+1}$. By part 2 of Lemma 2, $\Phi_{p i v}(g)$ goes to minus infinity as $g$ goes to minus infinity. Therefore, we can define $g^{\prime}$ such that

$$
\Phi_{p i v}(g)<v_{2 m-k+1}, \quad \text { for } g<g^{\prime} .
$$

By part 3 of Lemma 2, $\phi_{i}\left(v_{2 m-k+1}\right)$ goes to minus infinity as $v_{i}$ goes to minus infinity. Therefore, there exists $\underline{v}$ such that, for $i \leq 2 m-k$, if $v_{i} \leq \underline{v}$ then $\phi_{i}\left(v_{2 m-k+1}\right) \leq g^{\prime}$. Under decision rule $k$, at least one agent from the group $\{1, \ldots, 2 m-k\}$ must vote for $\beta$ in order for $\beta$ to be adopted by the group. Therefore, $\phi_{p i v}\left(v_{2 m-k+1}\right) \leq g^{\prime}$. This, together with the displayed inequality above, implies that

$$
\Phi_{p i v}\left(\phi_{p i v}\left(v_{2 m-k+1}\right)\right)<v_{2 m-k+1} .
$$

Recall from the proof of equilibrium existence in Proposition 1 that for any $x<v_{1}$, $\Phi_{\text {piv }}\left(\phi_{\text {piv }}(x)\right)-x$ is strictly positive. Therefore, there exists $\hat{G} \in\left(x, v_{2 m-k+1}\right)$ that satisfies $\Phi_{\text {piv }}\left(\phi_{\text {piv }}(\hat{G})\right)-\hat{G}=0$. Because $\hat{G}<v_{2 m-k+1}$, we have $\hat{G}<v_{i}$ for all $i \geq 2 m-k$. All these $k-1$ agents prefer $\beta$ to $\alpha$ when $\alpha$ is adopted. 
Proof of Proposition 7. We prove the proposition for the case where the lower cutoff is constrained. The proof of the other case is similar.

If $\phi_{p i v}(G) \geq v_{m}$, then $\phi_{p i v}^{c}(G)=\min \left\{\phi_{p i v}(G), v_{m}\right\}=v_{m}$. Since $\Phi_{p i v}\left(v_{m}\right)>v_{m}$, we have $\Phi_{\text {piv }}^{c}\left(v_{m}\right)=\Phi_{p i v}\left(v_{m}\right)$. This proves that $\left(v_{m}, \Phi_{p i v}\left(v_{m}\right)\right)$ is an unequal fixed point of $\left(\phi_{p i v}^{c}, \Phi_{p i v}^{c}\right)$ if $\phi_{p i v}\left(\Phi_{p i v}\left(v_{m}\right)\right) \geq v_{m}$ and $\Phi_{p i v}\left(v_{m}\right)>v_{m}$.

Conversely, $\left(v_{m}, \Phi_{p i v}\left(v_{m}\right)\right)$ cannot be an unequal fixed point of $\left(\phi_{p i v}^{c}, \Phi_{p i v}^{c}\right)$ if either $\phi_{p i v}\left(\Phi_{p i v}\left(v_{m}\right)\right)<v_{m}$ or $\Phi_{p i v}\left(v_{m}\right) \leq v_{m}$. If $\phi_{p i v}\left(\Phi_{p i v}\left(v_{m}\right)\right)<v_{m}$, then $\phi_{p i v}^{c}\left(\Phi_{p i v}\left(v_{m}\right)\right)=$ $\phi_{p i v}\left(\Phi_{p i v}\left(v_{m}\right)\right)<v_{m}$. Recall that $\Phi_{p i v}\left(v_{m}\right) \geq v_{m}$ for all $k_{D} \geq m$. Hence, $\Phi_{p i v}\left(v_{m}\right)=v_{m}$ if $\Phi_{\text {piv }}\left(v_{m}\right) \leq v_{m}$. But then $\left(v_{m}, v_{m}\right)$ is not unequal.

Finally, $\Phi_{p i v}\left(v_{m}\right)>v_{m}$ holds only when $k_{D}>m$. For any rule $k_{D}>m$,

$$
\phi_{p i v}\left(\Phi_{\text {piv }}\left(v_{k_{D}}\right)\right)=\phi_{\text {piv }}\left(v_{k_{D}}\right)<v_{k_{D}} .
$$

It follows that if $\left(v_{m}, \Phi_{p i v}\left(v_{m}\right)\right)$ is an unequal fixed point of $\left(\phi_{p i v}^{c}, \Phi_{p i v}^{c}\right)$, then

$$
\phi_{p i v}\left(\Phi_{p i v}\left(v_{m}\right)\right) \geq \phi_{p i v}^{c}\left(\Phi_{p i v}\left(v_{m}\right)\right)=v_{m} .
$$

Hence, $\left(\phi_{p i v}, \Phi_{\text {piv }}\right)$ must have an unequal fixed point $(\hat{g}, \hat{G})$ with $\hat{g} \in\left[v_{m}, v_{k_{D}}\right)$. At this equilibrium outcome, at most $m$ agents prefers $\beta$ at $\hat{g}$. Furthermore, since $\Phi_{p i v}^{c}(g) \geq \Phi_{p i v}(g)$ and $\phi_{p i v}^{c}(G) \leq \phi_{p i v}(G)$, the same reasoning as in the proof of Proposition 4 establishes that the waiting region is wider under the constrained two-stage decision process.

Proof of Proposition 8. Suppose $(\hat{g}, \hat{G})$ is an equilibrium in the one-stage deliberation game with rule $k=k_{D}$. Suppose agent $j$ is pivotal for the upper cutoff in this equilibrium. For any fixed $g$, the best response of agent $j$ diverges to infinity as $r_{j}$ goes to zero. Because $\lim _{r_{j} \rightarrow 0} R_{1}=0$ and $\lim _{r_{j} \rightarrow 0} R_{2}=1$, we have

$$
\lim _{r_{j} \rightarrow 0} f\left(r_{j}, G-g\right)=\lim _{r_{j} \rightarrow 0} \log \frac{R_{2} e^{R_{1}(G-g)}-R_{1} e^{R_{2}(G-g)}}{R_{2}-R_{1}}=0 .
$$

The sign of $\partial u_{j} / \partial G$ depends only on the sign of $v_{j}-g-f\left(r_{j}, G-g\right)$. This implies that, for any $g<v_{j}, \lim _{r_{j} \rightarrow 0} \partial u_{j} / \partial G>0$ for any $G$. Thus $G$ must increase without bound as $r_{j}$ goes to 0 . A similar argument establishes that $g$ must decrease without bound as the pivotal agent for the lower cutoff has a discount rate that goes to 0 . Finally, since $f(r, G-g)$ is monotone increasing in $r$ (proof of Lemma 2, part 3), there exists $\underline{r}$ such that $\hat{G}>v_{2 m-1}$ and $\hat{g}<v_{1}$ whenever $r_{i} \leq \underline{r}$ for all agents $i$.

Next, we show that such $(\hat{g}, \hat{G})$ is an equilibrium outcome of the two-stage decision process with any decision rule $k_{d}$. We first note that it is an equilibrium for each agent $i$ to vote for $\alpha$ if $\theta \geq v_{i}$ and to vote for $\beta$ otherwise, because $\alpha$ is preferred to flipping a coin (which is in turn preferred to $\beta$ ) whenever $\theta \geq v_{i}$, while $\beta$ is preferred to flipping a coin 
(which is in turn preferred to $\alpha$ ) whenever $\theta<v_{i}$. Given such equilibrium second-stage strategy, and given the fact that $\hat{G}>v_{2 m-1}, \alpha$ will be adopted with unanimous support for any $k_{d}$ if the belief reaches the upper cutoff. Likewise, $\beta$ will be adopted with unanimous support for any $k_{d}$ if the belief reaches the lower cutoff.

In the first stage, suppose each agent $i$ adopts the strategy $\left(g_{i}, G_{i}\right)=\left(\phi_{i}(\hat{G}), \Phi_{i}(\hat{g})\right)$. As $r_{i}$ goes to 0 , we have $G_{i}>v_{2 m-1}$ for every $i$. Given this first-stage strategy, the span of control $I^{G}\left(\sigma_{-i}\right)$ for any agent $i$ over the upper cutoff is above $v_{2 m-1}$. Similarly, the span of control for any agent over the lower cutoff is below $v_{1}$. For any decision rule $k_{d}$ which is non-unanimous, it is not feasible for any agent to unilaterally deviate to obtain an indecisive outcome, given the strategy profile of other agents. Furthermore, it is not profitable to unilaterally deviate to delay or hasten the adoption of either alternative, because $(\hat{g}, \hat{G})$ solves the constrained maximization problem 1 for the one-stage problem. If the decision rule $k_{d}$ is unanimous, it is feasible for an agent to deviate by changing his thresholds in the first stage and withholding his support for an alternative in the second stage to obtain an indecisive outcome. But doing so is worse than just changing the thresholds in the first stage and voting sincerely in the second stage, because an indecisive outcome is worse than $\alpha$ for any $\theta \in I^{G}\left(\sigma_{-i}\right)$, and is worse than $\beta$ for any $\theta \in I^{g}\left(\sigma_{-i}\right)$. Since the latter deviation is unprofitable, deviation to force flipping a coin cannot be profitable for any belief within an agent's span of control. 


\section{References}

Albrecht, James, Axel Anderson, and Susan Vroman (2010), "Search by Committee," Journal of Economic Theory, 145, 1386-1407.

Austen-Smith, David and Timothy Feddersen (2005), "Deliberation and Voting Rules," In Social Choice and Strategic Decisions: Essays in Honor of Jeffrey S. Banks, edited by David Austen-Smith and John Duggan, Berlin: Springer-Verlag.

Austen-Smith, David and Timothy Feddersen (2006), "Deliberation, Preference Uncertainty, and Voting Rules," American Political Science Review, 100, 209-218.

Baldwin, John and Michael McConville (1980), "Juries, Foremen, and Verdicts," British Journal of Criminology, 20(1), 34-44.

Blinder, Alan S. and John Morgan (2008), "Leadership in Groups: A Monetary Policy Experiment," International Journal of Central Banking, 4(4), 117-150.

Bognar, Katalin, Moritz Meyer-ter-Vehen, and Lones Smith (2015), "A Conversational War of Attrition," mimeo.

Brown, Stephen A. (1997), Revolution at the Checkout Counter, Cambridge: Harvard University Press.

Chiao, Benjamin, Josh Lerner, and Jean Tirole (2007), “The Rules of Standard-Setting Organizations: An Empirical Analysis," RAND Journal of Economics, 38, 905-930.

Compte, Olivier and Philippe Jehiel (2010), "Bargaining and Majority Rules: A Collective Search Perspective," Journal of Political Economy, 118, 189-221.

Coughlan, Peter (2000), "In Defense of Unanimous Jury Verdicts: Mistrials, Communication, and Strategic Voting," American Political Science Review, 94, 375-393.

Cox, David R. and H. D. Miller (1965), The Theory of Stochastic Processes, London: Chapman and Hall.

De Groot, M. H. (1970), Optimal Statistical Decisions, New York: McGraw-Hill.

Dixit, Avinash (1993), The Art of Smooth Pasting, Chur: Harwood Academic Publishers.

Dvoretsky, Aryeh, Jack Keifer, and Jacob Wolfowitz (1953), "Sequential Decision Problems for Processes with Continuous Time Parameter: Testing Hypothesis," Annals of Mathematical Statistics, 24, 254-264.

Ellsworth, Phoebe C. (1989), "Are Twelve Heads Better than One?" Law and Contemporary Problems, 52(4), 205-224. 
Eso, Peter and Yuk-Fai Fong (2008), "Wait and See: A Theory of Communication Over Time," mimeo.

Farrell, Joseph and Garth Saloner (1988), "Coordination through Committees and Markets," RAND Journal of Economics, 19, 235-252.

Farrell, Joseph and Timothy Simcoe (2012), "Choosing the Rules for Consensus Standardization," RAND Journal of Economics, 43, 235-252.

Feddersen, Timothy and Wolfgang Pesendorfer (1996), "The Swing Voter's Curse," American Economic Review, 86, 408-424.

Feddersen, Timothy and Wolfgang Pesendorfer (1998), "Convicting the Innocent: the Inferiority of Unanimous Jury Verdicts Under Strategic Voting," American Political Science Review, 92(1), 23-35.

Fishkin, James (2009), When the People Speak: Deliberative Democracy and Public Consultation, Oxford: Oxford University Press.

Gerardi, Dino and Leeat Yariv (2007), "Deliberative Voting," Journal of Economic Theory, 134, 317-338.

Gerardi, Dino and Leeat Yariv (2008), "Information Acquisition in Committees," Games and Economic Behavior, 62, 436-459.

Goeree, Jacob K. and Leeat Yariv (2011), “An Experimental Study of Collective Deliberation," Econometrica, 89(3), 893-921.

Gul, Faruk and Wolfgang Pesendorfer (2012), "War of Information," Review of Economic Studies, 79, 707-734.

Guttmann, Amy and Dennis Thompson (2004), Why Deliberative Democracy? Princeton: Princeton University Press.

Hamilton, Alexander, James Madison, and John Jay (1982), The Federalist Papers, New York: Bantom Books.

Hannaford, Paula L., Valerie P. Hans, Nicole L. Mott, and Thomas G. Musterman (2000), "The Timing of Opinion Formation by Jurors in Civil Cases: an Empirical Examination," Tennessee Law Review, 67, 627-652.

Hans, Valerie P. (2007), "Deliberation and Dissent: 12 Angry Men versus the Empirical Reality of Juries," Chicago-Kent Law Review, 82, 579-589.

Hastie, Reid, Steven D. Penrod, and Nancy Pennington (1983), Inside the Jury, Cambridge: Harvard University Press. 
Henry, Emeric and Marco Ottaviani (2014), "Research and the Approval Process," mimeo.

Kalven, Harry and Hand Zeisel (1966), The American Jury, Little Brown, Boston.

Levinson, Marc (2006), The Box, Princeton: Princeton University Press.

Li, Hao and Wing Suen (2009), "Decision-making in Committees," Canadian Journal of Economics, 42(2), 359-392.

Meirowitz, Adam (2006), "Designing Institutions to Aggregate Private Beliefs and Values," Quarterly Journal of Political Science, 1(4), 373-392.

Messner, Matthias and Mattias Polborn (2012), "The Option to Wait in Collective Decisions," Journal of Public Economics, 96, 524-540.

Mikhalevich, V.S. (1958), "Bayesian Choice Between Two Hypotheses on the Mean Value of a Normal Process," Visnyk Kyivs'koho Universytetu, Kiev, 1(1), 101-104.

Moldovanu, Benny and Xianwen Shi (2013), "Specialization and Partisanship in Search Committees," Theoretical Economics, 8, 751-774.

Moscarini, Giuseppe and Lones Smith (2001), "The Optimal Level of Experimentation," Econometrica, 69, 1629-1644.

Murphy, Beth and Robert G. Boatright (1999a), "Behind Closed Doors: A Resource Manual to Improve Jury Deliberation," Judicature, 83(2), 52-57.

Murphy, Beth and Robert G. Boatright (1999b), "How Judges Can Help Deliberating Juries: Using the Guide for Jury Deliberations," Court Review, 36(2), 38-45.

Persico Nicola (2004), “Committee Design with Endogenous Information," Review of Economic Studies, 71(1), 165-194.

Riker, William (1982), Liberalism against Populism, Prospect Heights: Waveland Press.

Shiryaev, Albert N. (1967) “Two Problems of Sequential Analysis," Cybernetics, 3(2), 6369.

Simcoe, Timothy (2012), "Standard Setting Committees: Consensus Governance for Shared Technology Platform," American Economic Review, 102(1), 305-336.

Steiner, Jurg (2012), The Foundations of Deliberative Democracy: Empirical Research and Normative Implications, Cambridge: Cambridge University Press.

Strulovici, Bruno (2010), “Learning while Voting: Determinants of Strategic Experimentation," Econometrica, 78, 933-971. 
Vidmar, Neil and Valerie P. Hans (2007), American Juries: the Verdict, New York: Prometheus Books.

Wald, Abraham (1947a), "Foundations of a General Theory of Sequential Decision Functions," Econometrica, 15(4), 279-313.

Wald, Abraham (1947b), Sequential Analysis, New York: Wiley.

Wilson, Alistair J. (2014), "Costly Communication in Groups: Theory and an Experiment,"mimeo. 